\title{
Finding the most variable stars in the Orion Belt with the All Sky Auto- mated Survey
}

\author{
José A. Caballero ${ }^{\star}$, M. Cornide, and E. de Castro \\ Departamento de Astrofísica y Ciencias de la Atmósfera, Facultad de Física, Universidad Complutense de Madrid, E- \\ 28040 Madrid, Spain
}

Received 05 Sep 2009, accepted 04 Jan 2010

Published online later

Key words stars: variables: general — stars: pre-main sequence — stars: oscillations — astronomical data bases: miscellaneous - Galaxy: open clusters and associations: Ori OB1 b

\begin{abstract}
We look for high-amplitude variable young stars in the open clusters and associations of the Orion Belt. We use public data from the ASAS-3 Photometric $V$-band Catalogue of the All Sky Automated Survey, infrared photometry from the 2MASS and IRAS catalogues, proper motions, and the Aladin sky atlas to obtain a list of the most variable stars in a survey area of side 5 deg centred on the bright star Alnilam ( $\epsilon$ Ori) in the centre of the Orion Belt. We identify 32 highly-variable stars, of which 16 had not been reported to vary before. They are mostly variable young stars and candidates (16) and background giants (8), but there are also field cataclysmic variables, contact binaries, and eclipsing binary candidates. Of the young stars, which typically are active Herbig Ae/Be and T Tauri stars with $\mathrm{H} \alpha$ emission and infrared flux excess, we discover four new variables and confirm the variability status of another two. Some of them belong to the well known $\sigma$ Orionis cluster. Besides, six of the eight giants are new variables, and three are new periodic variables.
\end{abstract}

(C) 0000 WILEY-VCH Verlag GmbH \& Co. KGaA, Weinheim

\section{Introduction}

The Ori OB 1 b subgroup association, mostly coincident with the asterism of the Orion Belt, contains a rich population of very young stars (e.g. Haro \& Moreno 1953; Walker 1969; Warren \& Hesser 1978; Brown et al. 1994; Sterzik et al. 1995; de Zeeuw et al. 1999; Briceño et al. 2001). The younges regions, including Alnitak ( $\zeta$ Ori), which illuminates the Flame Nebula (NGC 2024), and $\sigma$ Ori, which illuminates the Horsehead Nebula (Barnard 33 and IC 434), are to the east of the Belt and have ages of 1 to $5 \mathrm{Ma}$. Here lies the $\sigma$ Orionis cluster, one of the most suitable sites for discovering and characterising substellar objects (Béjar et al. 1999; Zapatero Osorio et al. 2002; Sherry et al. 2004; Caballero et al. 2007). The population of stars surrounding Alnilam ( $\epsilon$ Ori) and Mintaka ( $\delta$ Ori), to the west of the Belt, are older (5-10 Ma) and spread over a wider area than the much denser $\sigma$ Orionis cluster (Caballero \& Solano 2008). Surrounding the Belt, there is another dispersed population of 10-20 Ma-old stars, mostly associated to the Ori OB1 a subgroup association (Jeffries et al. 2006; Caballero 2007) and its agglomerates, such as the 25 Orionis group or the $\eta$ Orionis overdensity (Briceño et al. 2007; Caballero \& Dinis 2008).

$\mathrm{H} \alpha$ and X-ray emission and infrared excess are the primary features for the identification of very young stars as those in the Orion Belt. Optical and near-infrared variability, cosmic lithium abundances, surrounding jets, or forbid-

\footnotetext{
* Corresponding author: e-mail: caballero@astrax.fis.ucm.es
}

den emission lines are other signposts used to confirm very young ages in stars. Photometric variability in young stars is mostly due to dark (cool) and bright (hot) spots and flare activity, which are related to the strengthened magnetic activy due to fast rotation, and interactions with circumstellar discs, such as intense, variable accretion episodes or occultations (e.g. classical works: Joy 1945; Walker 1956; Herbig 1977 - theoretical works: Koenigl 1991; Collier Comeron \& Campbell 1993; Shu et al. 1994 - modern observational works: Bouvier et al. 1993; Herbst et al. 1994; Thé et al. 1994; Hartmann \& Kenyon 1996; Hamilton et al. 2001; Eiroa et al. 2002).

The Orion Nebula Cluster in the Ori OB1 d subgroup association has been a traditional target for studying the photometric variability of young stars (Jones \& Walker 1988; Carpenter et al. 2001; Herbst et al. 2002; Stassun et al. 2006; Parihar et al. 2009), but the surveys for variability in the Orion Belt are relatively scarce. The most thorough variability surveys in the Ori OB1 b association have been aimed at the low-mass pre-main sequence stars and brown dwarfs (Bailer-Jones \& Mundt 2001; Zapatero Osorio et al. 2003; Caballero et al. 2004, 2006; Scholz \& Eislöffel 2004, 2005; Briceño et al. 2005; Scholz et al. 2009), while the brightest variable stars were first recognised as early as the beginning of the 20th century and have not received quite attention. Only a few (multiple) young stars of important astrophysical interest had been extensively monitored, such as VV Ori (Miller Barr 1904; Struve \& Luyten 1949; Terrell et al. 2007), $\sigma$ Ori E (Walborn \& Hesser 1976; Landstreet \& Borra 1978), or Mintaka AE-D (Stebbins 1915; Koch 


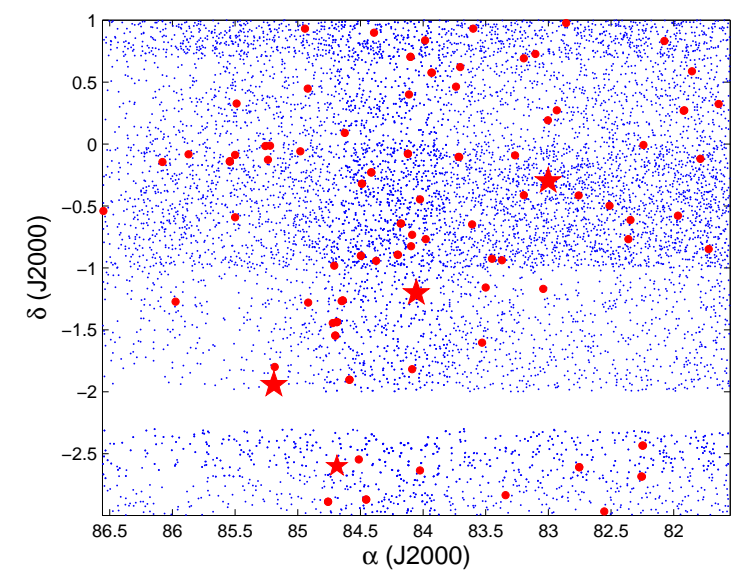

Fig. 1 Spatial location of the 10029 ASAS sources in the studied area with light curves with more than 40 data points, marked with (blue) small dots. North is up, east is to the left. The 100 ASAS sources with possible variable light curves, the three Orion Belt supergiant stars (Alnitak, Alnilam, and Mintaka, from east to west), and $\sigma$ Ori are marked with (red) filled circles, big stars, and a small star, respectively. Note the blank strip where ASAS did not survey (between Alnitak and $\sigma$ Orionis' declinations) and the overlapping between different ASAS pointings (at about the Mintaka's declination).

\& Hrivnak 1981). Many of the intermediate-mass T Tauri stars in the Orion Belt with variability denominations (e.g. BG Ori, V505 Ori) were compiled by Fedorovich (1960) and have never been monitored again. After a century of photometric searches in the region, there are still serendipitous discoveries of bright, UX Orionis-, A-type, variable stars (Caballero et al. 2008).

Our aim is to find the most variable stars in the Orion Belt, giving especial emphasis to the young Herbig Ae/Be (HAeBe) and T Tauri stars. To accomplish that, we have used the ASAS-3 Photometric $V$-band Catalogue of the All Sky Automated Survey (ASAS) $\sqrt{1}$, which is "a low cost project dedicated to constant photometric monitoring of the whole available sky" (Pojmański 1997, 2002).

\section{Analysis}

First, we compiled 63276 ASAS light curves of objects in a square of side $5.0 \mathrm{deg}$ centred on Alnilam; see Fig. 1. We used the default parameters $N>4$ data points and $r<$ $15 \operatorname{arcsec}(N$ is the number of data points within a circle of radius $r$ centred on the coordinates of an ASAS source). If not for certain blank strip, the surveyed area would have been $25 \mathrm{deg}^{2}$. To maximise the scientific return and minimise possible further troubles with light curves of poor quality, we discarded all ASAS light curves with $N \leq 40$ data points. It left 10029 light curves $(16 \%)$ with $N>40$

1 http://www.astrouw.edu.pl/asas/.

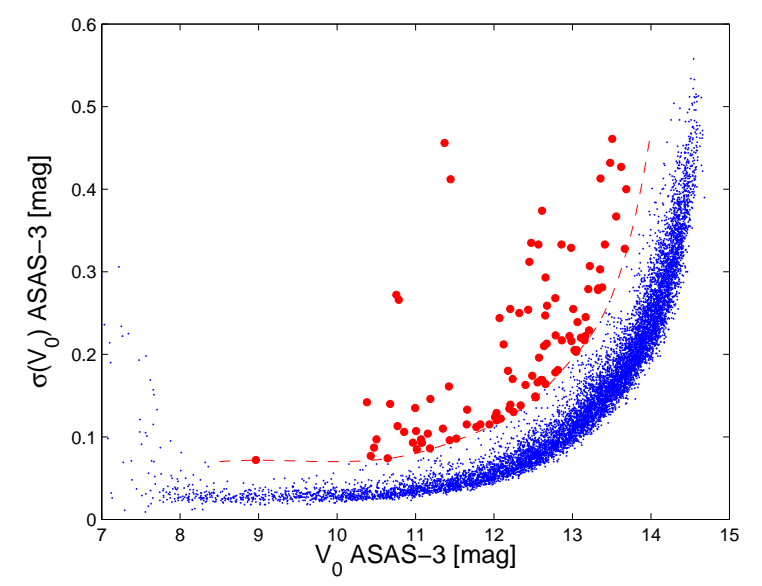

Fig. 2 Diagram showing $\sigma\left(V_{0}\right)$ vs. $\overline{V_{0}}$. ASAS sources above the dashed line are variable star candidates in this work. X Ori is out of limits $\left(\overline{V_{0}} \sim 12.7 \mathrm{mag}, \sigma\left(V_{0}\right) \sim\right.$ $1.4 \mathrm{mag})$.

for next phases of the analysis. The ASAS data covered almost six years during the first decade of the 21 st century.

Of the five available apertures $(0,1,2,3,4)$, the " 0 " aperture photometry seemed to provide better data (i.e. less dispersion and number of grade " $C$ " and " $D$ " data points - probably useless data or without photometric value at all due to bad pixels, frame-edge and bright star/planet proximity, or excessive faintness). According to Pojmański (2002), the five apertures correspond to diameter sizes of the aperture photometry from 2 to 6 pixels; the " 0 " aperture corresponds to the narrower photometry (Pojmański, priv. comm.). From this point on, we used only the Johnson $V$ magnitude with the ASAS " 0 " aperture photometry, which we indicate with the symbol $V_{0}$.

Fig. 2 illustrates the selection of variable stars. It is a $\sigma\left(V_{0}\right)$ vs. $\overline{V_{0}}$ diagram in which the variable star candidates have larger values of standard deviation $\sigma\left(V_{0}\right)$ with respect to non-variable stars of the same average magnitude $\overline{V_{0}}$. ASAS stars brighter than $\overline{V_{0}} \approx 8.5$ mag are affected by saturation in the detector and were not considered. The lower envelope at the non-saturated, brightest end of the cloud of data points in the diagram indicates the best photometric accuracy of the system, which is of about $0.02 \mathrm{mag}$. The obvious rise of the lower envelope at faint magnitudes is a combination of the standard deviations (root-mean-squares) from Poisson noise in the targets and from sky noise in the photometric aperture (e.g. Irwin et al. 2007). The line used as the selection criterion is the lower envelope shifted to larger $\sigma\left(V_{0}\right)$ s by magnitude-dependent amounts (roughly $0.06 \mathrm{mag}$ at $\overline{V_{0}} \sim 8.5-11.5 \mathrm{mag}, 0.12 \mathrm{mag}$ at $\overline{V_{0}} \sim 12.5 \mathrm{mag}$, and $0.18 \mathrm{mag}$ at $\overline{V_{0}} \sim 13.5 \mathrm{mag}$ ). This choice was relatively arbitrary: more or less restrictive selection criteria would have given significantly less or more variable star candidates, respectively. Thus, the used selection criterion was a compromise to minimise the number of false positives (variable star candidates that actually do not vary) and maximise 
Table 1 Identified variables in the Orion Belt.

\begin{tabular}{|c|c|c|c|c|c|c|c|c|c|c|}
\hline No. & $\begin{array}{l}\alpha^{2 \mathrm{MASS}} \\
(\mathrm{J} 2000)\end{array}$ & $\begin{array}{l}\delta^{2 \mathrm{MASS}} \\
(\mathrm{J} 2000)\end{array}$ & $\begin{array}{c}\overline{V_{0}} \\
{[\mathrm{mag}]}\end{array}$ & $\begin{array}{l}\sigma\left(V_{0}\right) \\
{[\mathrm{mag}]}\end{array}$ & $\begin{array}{c}\overline{\delta V_{0}} \\
{[\mathrm{mag}]}\end{array}$ & $N_{\mathrm{obs}}^{\star}$ & $\begin{array}{l}\text { Spectral } \\
\text { type }\end{array}$ & Name & Class & Variable \\
\hline 01 & 052859.57 & -033352.3 & 13.621 & 0.412 & 0.055 & 190 & $\ldots$ & V1159 Ori & Dwarf nova & Known \\
\hline 03 & 053101.46 & -03 2324.3 & 12.492 & 0.341 & 0.056 & 294 & $\ldots$ & IRAS 05285-0325 & Giant & New \\
\hline 06 & 053322.45 & -030955.7 & 11.185 & 0.085 & 0.032 & 207 & $\ldots$ & No. 06 & Unknown & New \\
\hline 07 & 053748.79 & -030748.7 & 10.780 & 0.101 & 0.021 & 286 & $\ldots$ & IRAS 05353-0309 & Giant & New \\
\hline 11 & 053209.94 & -024946.8 & 11.581 & 0.524 & 0.032 & 191 & F5-8Vpe & RY Ori & $\mathrm{HAeBe}$ & Known \\
\hline 12 & 053833.68 & -024414.2 & 12.885 & 0.368 & 0.054 & 187 & $\mathrm{~K} 4 \mathrm{e}$ & TX Ori & $\mathrm{T}$ Tauri & Known \\
\hline 13 & 053825.87 & -024351.2 & 13.059 & 0.356 & 0.054 & 142 & $\mathrm{~K} 3 \mathrm{e}$ & TY Ori & $\mathrm{T}$ Tauri & Known \\
\hline 14 & 054354.26 & -024335.4 & 11.628 & 0.135 & 0.032 & 222 & $\ldots$ & 2M054354-0243.6 & Contact binary & Known \\
\hline 15 & 053939.99 & -024309.7 & 13.246 & 0.246 & 0.054 & 197 & $\ldots$ & RW Ori & $\mathrm{T}$ Tauri & Known \\
\hline 18 & 053848.04 & -022714.2 & 13.340 & 0.295 & 0.053 & 224 & $\mathrm{~K} 7 \mathrm{e}+\mathrm{M}:$ & Mayrit 528005 AB & $\mathrm{T}$ Tauri & New \\
\hline 21 & 053620.91 & -021057.6 & 12.607 & 0.192 & 0.056 & 252 & F3e & PQ Ori & $\mathrm{HAeBe}$ & Known \\
\hline 29 & 053738.67 & -014616.6 & 12.752 & 1.441 & 0.054 & 179 & M8-9III: & X Ori & Giant & Known \\
\hline 30 & 053756.59 & -014050.3 & 10.734 & 0.250 & 0.021 & 273 & $\ldots$ & IRAS 05354-0142 & Giant & New \\
\hline 33 & 053002.89 & -013005.3 & 12.786 & 0.263 & 0.056 & 176 & $\ldots$ & No. 33 & Unknown & New \\
\hline 36 & 053425.82 & -012106.5 & 12.667 & 0.325 & 0.055 & 170 & $\ldots$ & V469 Ori & $\mathrm{T}$ Tauri & Known \\
\hline 41 & 052653.53 & -010902.3 & 13.478 & 0.480 & 0.057 & 144 & $\ldots$ & Kiso A-0903 135 & T Tauri? & New \\
\hline 43 & 053759.04 & -010552.8 & 13.554 & 0.371 & 0.054 & 200 & $\ldots$ & V993 Ori & T Tauri? & Known \\
\hline 46 & 053126.41 & -005833.1 & 10.454 & 0.076 & 0.021 & 192 & F0 & HD 290509 & Unknown & New \\
\hline 48 & 053945.65 & -005550.9 & 10.934 & 0.075 & 0.021 & 203 & $\ldots$ & GSC 04767-00071 & $\mathrm{T}$ Tauri? & Known \\
\hline 51 & 053624.29 & -004212.0 & 13.399 & 0.388 & 0.056 & 227 & $\mathrm{~K} 3 \mathrm{e}$ & PU Ori & $\mathrm{T}$ Tauri & Known \\
\hline 53 & 053448.91 & -003716.7 & 13.384 & 0.298 & 0.056 & 178 & $\ldots$ & V472 Ori & T Tauri? & Known \\
\hline 54 & 052724.67 & -003511.2 & 11.031 & 0.134 & 0.033 & 184 & $\ldots$ & IRAS 05248-0037 & Giant & New \\
\hline 55 & 053543.27 & -003436.7 & 12.411 & 0.278 & 0.057 & 246 & $\mathrm{~K} 4 \mathrm{e}$ & StHA 48 & $\mathrm{~T}$ Tauri & New \\
\hline 60 & 052634.28 & -001927.2 & 12.353 & 0.137 & 0.056 & 181 & $\ldots$ & No. 60 & Giant & New \\
\hline 65 & 053628.55 & +00 0445.7 & 11.987 & 0.131 & 0.056 & 257 & $\ldots$ & No. 65 & Unknown & New \\
\hline 66 & 054329.25 & +000458.9 & 11.459 & 0.513 & 0.032 & 202 & F0? & GT Ori & Giant & Known \\
\hline 70 & 054418.80 & +000840.4 & 8.906 & 0.061 & 0.019 & 204 & A7IIIe & V351 Ori & $\mathrm{HAeBe}$ & Known \\
\hline 72 & 054611.86 & +00 3225.9 & 13.390 & 0.439 & 0.053 & 167 & $\ldots$ & VSS VI-32 & Unknown & New \\
\hline 73 & 054159.81 & +00 3527.1 & 12.698 & 0.435 & 0.053 & 185 & $\ldots$ & No. 73 & Unknown & New \\
\hline 74 & 053642.64 & +00 3834.3 & 11.010 & 0.101 & 0.033 & 254 & $\mathrm{~A} 2$ & HD 290625 & $\mathrm{HAeBe}$ & New \\
\hline 75 & 053347.85 & +00 5536.3 & 10.433 & 0.166 & 0.021 & 181 & $\ldots$ & IRAS $05312+0053$ & Giant & New \\
\hline 78 & 052817.85 & +01 1006.1 & 11.219 & 0.165 & 0.033 & 173 & $\mathrm{~F} 8-\mathrm{K} 0 \mathrm{e}$ & StHA 40 & $\mathrm{~T}$ Tauri & Known \\
\hline
\end{tabular}

the number of actual variable stars, but always keeping a maneagable number of objects to be followed up.

There were 100 ASAS light curves above the selection criterion in Fig. 2. We were able to identify the 2MASS (Skrutskie et al. 2006) and USNO-B1 (Monet et al. 2003) possible stellar counterparts of 99 of them. The unidenfied ASAS source was 054200-0154.7 ( $V_{0}=13.167 \mathrm{mag}, \sigma\left(V_{0}\right)$ $=0.245 \mathrm{mag}, \mathrm{N}_{\mathrm{obs}}=49$ ), which is actually a bright spot in NGC 2024. For the visual identification, we used the Aladin interactive sky atlas (Bonnarel et al. 2000). The actual number of stars with light curves was 78 , because there were 21 variable star candidates with two corresponding light curves. The separated datasets (with identical associated coordinates and magnitudes within error bars) correspond to the same star in different observed fields (Pojmański 2002).

We revisited the ASAS data for the 78 stars by retrieving all data points with quality grades " $A$ " and " $B$ " in a circle of radius $r=15$ arcsec centred on the stars, and merging them into a unique light curve. "A" and "B"quality grades, which are tabulated by the ASAS catalogue and derived from the average photometric quality of the frame for each aperture, correspond to the best and mean data, respectively2. Because of the large ASAS pixel size (of about $15 \mu \mathrm{m}$ ) and typical full width at half maximum, of no less than 1.4 pixels (Pojmański 2002), we discarded all visual binaries of roughly the same brightness separated by less than about 20 arcsec. Besides, we also discarded relatively faint variable candidates at less than 1 arcmin to very bright stars, including Alnilam, Mintaka, and $\sigma$ Ori, which are seen by the naked eye and lead to a high sky background. After these removals, we kept 32 variable stars whose light curves were likely not contaminated by other stars. The 32 light curves are shown in Figs. A1 to A4

The identification number, 2MASS coordinates, average $\overline{V_{0}}$ magnitude, standard deviation $\sigma\left(V_{0}\right)$, mean error of magnitude $\overline{\delta V_{0}}$, and number of finally used light curve data points, $N_{\mathrm{obs}}^{\star}$, of the 32 identified variable stars are listed in Table 1. The ratios between the standard deviations and mean errors vary between $\sim 2.5$ and 27 ; the expected ratio

\footnotetext{
2 The quality grades "A" and "B" of a new ASAS frame depends on the average dispersion between 'old' and 'new' magnitudes for stars in the range 8-11 mag (Pojmański, priv. comm.).
} 


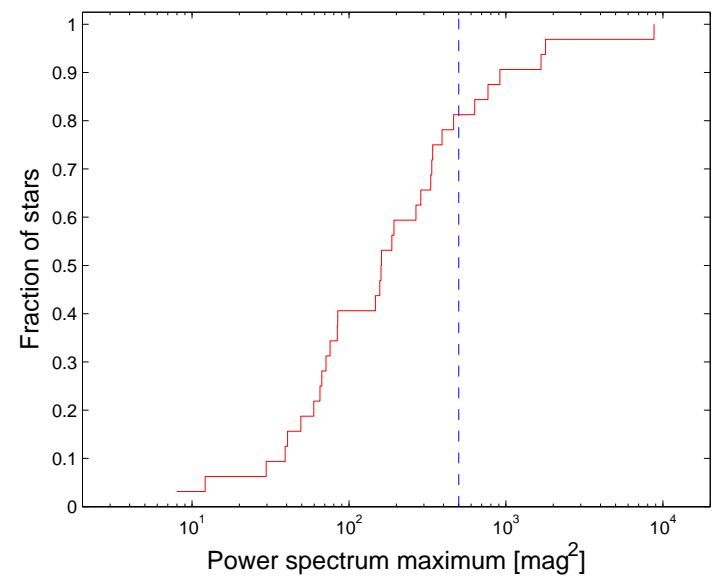

Fig. 3 Cumulative distribution function of power spectrum maxima of the light curves of the 32 identified variables in the Orion Belt. Stars to the right of the dashed vertical line at power spectrum of $500 \mathrm{mag}^{2}$ are classified here as probable periodic variable stars.

for a non-variable star should be lower than 2 (e.g. BailerJones \& Mundt 2001). In Table 1, we also provide the spectral type from the literature (if it has ever been given), name, variability class (see Section 3), and if the variability status was known or not. For those stars that have never been mentioned in the literature, we use the nomenclature "No. $X$ ", where $X$ is the identification number.

For a typical number of light curve data points of $N_{\mathrm{obs}}^{\star} \sim$ 200 and a temporal coverage of almost six years, one may derive that there was an ASAS visit every $\sim 11 \mathrm{~d}$ for each of the 32 stars. Actually, the typical time interval between consecutive ASAS visits was shorter because there were long non-observing gaps without data. The median time interval between consecutive ASAS visits was 2.0-3.0d, depending on the star. Approximately 15-20, 20-33, 65-85, and 85$95 \%$ of the time intervals were shorter than $1,2,5$, and $10 \mathrm{~d}$, respectively, and only in four cases, coincident with the four Austral summers, the time intervals were longer than $100 \mathrm{~d}$.

We performed a rutinary time-series analysis on the 32 light curves by applying the Scargle (1982) procedure. Fig. 3] shows the cumulative distribution function of the measured maxima of power spectrum. Roughly $85 \%$ of the stars have power spectrum maxima lower than $500 \mathrm{mag}^{2}$, where there seems to be an inflection point in the cumulative distribution function. The other $15 \%$ (6) of the stars with larger power spectrum maxima are given in Table 2 and were classified by us as probable periodic variable stars. We provide periods for two previously known periodic variable stars (V1159 Ori and X Ori) consistent with those in the literature, and found probable periods for two previously unknwon variable giant stars (with IRAS denomination), but failed to find any reliable periodicty for RY Ori and GT Ori. The six of them will be studied in detail in Section 3 .

We performed a compilation of additional infrared data by collecting $J H K_{\mathrm{s}}$ magnitudes from 2MASS and IRAS
Table 2 Identified variables with power spectrum maxima larger than $500 \mathrm{mag}^{2}$.

\begin{tabular}{lccll}
\hline \hline No. & $\begin{array}{c}\text { Power } \\
{\left[\mathrm{mag}^{2}\right]}\end{array}$ & $\begin{array}{c}P \\
{[\mathrm{~d}]}\end{array}$ & Name & Remarks \\
\hline 01 & 767.19 & 44.345 & V1159 Ori & $P=44.2-46.8 \mathrm{~d}$ \\
03 & 1785.4 & 78.226 & IRAS 05285-0325 & New periodic \\
11 & 632.39 & 600.38 & RY Ori & No clear peak \\
29 & 8779.7 & 416.05 & X Ori & $P=424.2 \pm 1.8 \mathrm{~d}$ \\
30 & 913.68 & 373.31 & IRAS 05354-0142 & New periodic \\
66 & 1671.2 & 920.32 & GT Ori & Pulsating \\
\hline
\end{tabular}

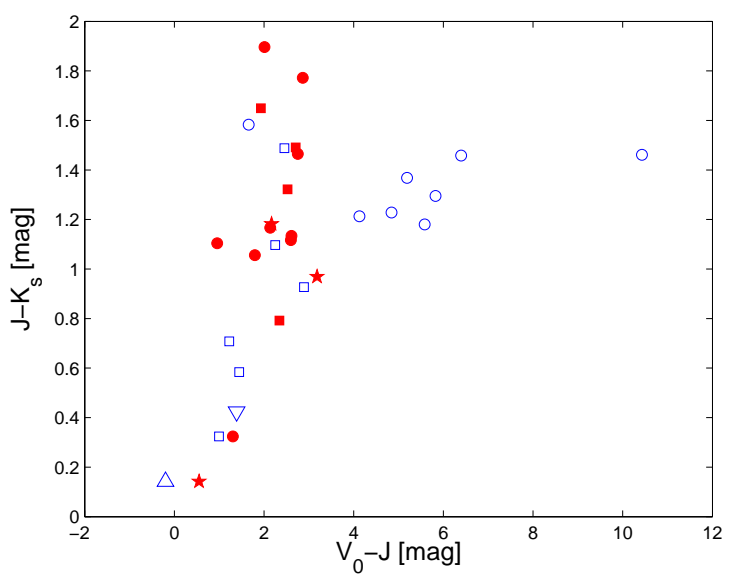

Fig. 4 Optical-infrared colour-colour diagram ( $J-K_{\mathrm{s}}$ vs. $\left.V_{0}-J\right)$. Filled (red) stars: new variable young stars; filled (red) circles: previously known variable young stars; filled (red) squares: variable young star candidates; open (blue) circles: variable giants; open (blue) up-triangle: dwarf nova (V1159 Ori); open (blue) down-triangle: contact binary (2M054354-0243.6); open (blue) squares: other new variable stars.

average non-colour corrected flux densities with qualities " 3 " or " 2 " (high and moderate qualities, respectively). While all the 32 stars have a near-infrared counterpart in the 2MASS catalogue, only 12 stars were identified in the IRAS catalogues (e.g. IRAS catalogue of point sources, version 2.0; IPAC 1986). Furthermore, only one star, the A7IIIe Herbig Ae/Be star V351 Ori, has high/moderate quality measurements at the four IRAS passbands at 12, 25, 60, and $100 \mu \mathrm{m}$. A colour-colour diagram combining ASAS optical and 2MASS near-infrared magnitudes is shown in Fig. 4.

Besides, we also collected proper motions from Tycho-2 (Høg et al. 2000), PPMX (Röser et al. 2008), and USNO-B1 for the 32 stars. Given that only a few of them have appreciable proper motions, we do not list them, but are mentioned in Section 3 when appropriated.

Finally, only 13 stars have variable star designations, and there were suspects of variability for other three stars. Besides, an important fraction of the targets have also been tabulated as variable stars of the Southern Hemisphere by Pojmański (2002), to which our work complements. Next, 
Table 3 Infrared photometry of identified variables.

\begin{tabular}{|c|c|c|c|c|c|c|c|}
\hline No. & $\begin{array}{c}J \\
{[\mathrm{mag}]}\end{array}$ & $\begin{array}{c}H \\
{[\mathrm{mag}]}\end{array}$ & $\begin{array}{c}K_{\mathrm{s}} \\
{[\mathrm{mag}]}\end{array}$ & $\begin{array}{l}F_{12} \\
\text { [Jy] }\end{array}$ & $\begin{array}{l}F_{25} \\
\text { [Jy] }\end{array}$ & $\begin{array}{l}F_{60} \\
{[\mathrm{Jy}]}\end{array}$ & $\begin{array}{c}F_{100} \\
{[\mathrm{Jy}]}\end{array}$ \\
\hline 01 & $13.817 \pm 0.027$ & $13.781 \pm 0.046$ & $13.675 \pm 0.050$ & $\ldots$ & $\ldots$ & $\ldots$ & $\ldots$ \\
\hline 03 & $6.097 \pm 0.019$ & $5.083 \pm 0.027$ & $4.639 \pm 0.021$ & $1.08 \pm 0.08$ & $0.39 \pm 0.04$ & $<3$ & $<20$ \\
\hline 06 & $8.295 \pm 0.034$ & $7.552 \pm 0.024$ & $7.368 \pm 0.026$ & $\ldots$ & $\ldots$ & $\ldots$ & $\ldots$ \\
\hline 07 & $5.590 \pm 0.021$ & $4.665 \pm 0.076$ & $4.222 \pm 0.033$ & $1.09 \pm 0.07$ & $0.47 \pm 0.08$ & $<0.9$ & $<30$ \\
\hline 11 & $9.444 \pm 0.023$ & $8.885 \pm 0.055$ & $8.277 \pm 0.031$ & $0.75 \pm 0.09$ & $0.71 \pm 0.09$ & $<2$ & $<20$ \\
\hline 12 & $10.131 \pm 0.026$ & $9.280 \pm 0.024$ & $8.666 \pm 0.024$ & $0.38 \pm 0.04$ & $0.50 \pm 0.07$ & $<0.7$ & $<40$ \\
\hline 13 & $10.445 \pm 0.027$ & $9.726 \pm 0.024$ & $9.311 \pm 0.028$ & $\ldots$ & $\ldots$ & $\ldots$ & $\ldots$ \\
\hline 14 & $10.240 \pm 0.026$ & $9.943 \pm 0.026$ & $9.816 \pm 0.021$ & $\ldots$ & $\ldots$ & $\ldots$ & $\ldots$ \\
\hline 15 & $10.647 \pm 0.027$ & $9.920 \pm 0.023$ & $9.530 \pm 0.019$ & $\ldots$ & $\ldots$ & $\ldots$ & $\ldots$ \\
\hline 18 & $10.156 \pm 0.023$ & $9.463 \pm 0.026$ & $9.187 \pm 0.019$ & $\ldots$ & $\ldots$ & $\ldots$ & $\ldots$ \\
\hline 21 & $11.303 \pm 0.022$ & $11.065 \pm 0.028$ & $10.979 \pm 0.023$ & $\ldots$ & $\ldots$ & $\ldots$ & $\ldots$ \\
\hline 29 & $2.322 \pm 0.292$ & $1.379 \pm 0.306$ & $0.861 \pm 0.346$ & $96 \pm 4$ & $49 \pm 7$ & $6.0 \pm 0.9$ & $<20$ \\
\hline 30 & $5.152 \pm 0.018$ & $4.608 \pm 0.076$ & $3.972 \pm 0.320$ & $2.0 \pm 0.2$ & $0.63 \pm 0.07$ & $<2$ & $<40$ \\
\hline 33 & $11.340 \pm 0.024$ & $10.878 \pm 0.025$ & $10.756 \pm 0.021$ & $\ldots$ & $\ldots$ & $\ldots$ & $\ldots$ \\
\hline 36 & $10.655 \pm 0.023$ & $9.647 \pm 0.030$ & $8.759 \pm 0.021$ & $\ldots$ & $\ldots$ & $\ldots$ & $\ldots$ \\
\hline 41 & $11.547 \pm 0.027$ & $10.652 \pm 0.027$ & $9.898 \pm 0.019$ & $\ldots$ & $\ldots$ & $\ldots$ & $\ldots$ \\
\hline 43 & $10.846 \pm 0.027$ & $9.955 \pm 0.024$ & $9.355 \pm 0.027$ & $\ldots$ & $\ldots$ & $\ldots$ & $\ldots$ \\
\hline 46 & $9.457 \pm 0.023$ & $9.247 \pm 0.023$ & $9.133 \pm 0.021$ & $\ldots$ & $\ldots$ & $\ldots$ & $\ldots$ \\
\hline 48 & $8.589 \pm 0.023$ & $7.933 \pm 0.055$ & $7.797 \pm 0.027$ & $\ldots$ & $\ldots$ & $\ldots$ & $\ldots$ \\
\hline 51 & $10.536 \pm 0.023$ & $9.551 \pm 0.033$ & $8.764 \pm 0.019$ & $<0.4$ & $0.38 \pm 0.05$ & $<3$ & $<20$ \\
\hline 53 & $10.860 \pm 0.022$ & $10.043 \pm 0.026$ & $9.538 \pm 0.019$ & $\ldots$ & $\ldots$ & $\ldots$ & $\ldots$ \\
\hline 54 & $5.206 \pm 0.017$ & $4.225 \pm 0.202$ & $3.911 \pm 0.258$ & $1.48 \pm 0.09$ & $0.49 \pm 0.04$ & $<5$ & $1.7 \pm 0.3$ \\
\hline 55 & $10.243 \pm 0.021$ & $9.509 \pm 0.033$ & $9.061 \pm 0.019$ & $\ldots$ & $\ldots$ & $\ldots$ & $\ldots$ \\
\hline 60 & $7.508 \pm 0.023$ & $6.596 \pm 0.029$ & $6.280 \pm 0.021$ & $\ldots$ & $\ldots$ & $\ldots$ & $\ldots$ \\
\hline 65 & $10.762 \pm 0.024$ & $10.447 \pm 0.025$ & $10.054 \pm 0.019$ & $\ldots$ & $\ldots$ & $\ldots$ & $\ldots$ \\
\hline 66 & $9.802 \pm 0.027$ & $9.043 \pm 0.025$ & $8.219 \pm 0.026$ & $0.87 \pm 0.13$ & $1.02 \pm 0.17$ & $<5$ & $<40$ \\
\hline 70 & $7.950 \pm 0.020$ & $7.504 \pm 0.040$ & $6.846 \pm 0.026$ & $1.16 \pm 0.07$ & $4.1 \pm 0.2$ & $26 \pm 3$ & $21 \pm 3$ \\
\hline 72 & $10.936 \pm 0.024$ & $10.046 \pm 0.023$ & $9.448 \pm 0.019$ & $\ldots$ & $\ldots$ & $\ldots$ & $\ldots$ \\
\hline 73 & $10.448 \pm 0.023$ & $9.818 \pm 0.023$ & $9.351 \pm 0.019$ & $\ldots$ & $\ldots$ & $\ldots$ & $\ldots$ \\
\hline 74 & $10.459 \pm 0.024$ & $10.342 \pm 0.024$ & $10.317 \pm 0.023$ & $\ldots$ & $\ldots$ & $\ldots$ & $\ldots$ \\
\hline 75 & $6.304 \pm 0.026$ & $5.375 \pm 0.027$ & $5.091 \pm 0.020$ & $0.47 \pm 0.03$ & $<0.2$ & $<0.4$ & $<20$ \\
\hline 78 & $9.422 \pm 0.023$ & $8.825 \pm 0.040$ & $8.366 \pm 0.018$ & $0.41 \pm 0.06$ & $0.67 \pm 0.06$ & $0.47 \pm 0.05$ & $<1.1$ \\
\hline
\end{tabular}

we classify the 32 variable stars based on spectroscopic, astrometric, and photometric criteria.

\section{Identified variable stars}

\subsection{HAeBe and T Tauri variable stars}

\subsubsection{Previously known variable young stars and candidates}

This class is mostly made of probable accreting $\mathrm{T}$ Tauri stars with intense $\mathrm{H} \alpha$ emission. Of the nine $\mathrm{H} \alpha$ emitter stars, three belong to the $\sigma$ Orionis cluster (TX Ori, TY Ori, RW Ori), three have been associated to the Alnilam and Mintaka young star populations (V469 Ori, PU Ori, V472 Ori) and three have not been linked to any young region in particular but are bright stars at the boundary between HAeBe and T Tauri stars (RY Ori, V351 Ori, StHa 40 - two of them may have edge-on discs). The true nature of the other three non$\mathrm{H} \alpha$ emitter stars (PQ Ori, V993 Ori, GSC 04767-00071) should be investigated with further spectroscopic analyses.
Besides, six of the stars in this class display X-ray emission, and other six, infrared flux excess. Only the three $\sigma$ Orionis members are known to simultaneously display $\mathrm{H} \alpha$ and $\mathrm{X}$ ray emissions and infrared flux excess.

RY Ori (No. 11). It is a well investigated, emission-line star of the Orion population at the boundary between HAeBe and T Tauri stars (e.g. Haro \& Moreno 1953; Herbig \& Bell 1988; Yudin \& Evans 1998; Eiroa et al. 2001; Mora et al. 2001; Hernández et al. 2004; Acke \& van den Ancker 2006). Its photometric variability was detected very early (Pickering 1904). According to Bibo \& Thé (1991), it is a UX Oritype star with a colour reversal in the colour-magnitude diagram, indicative of an edge-on disc. The disc of RY Ori s also detected because of its long-time-known infrared excess (Cohen 1973; Glass \& Penston 1974; Weintraub 1990; Weaver \& Gordon 1992). Its photometric variability in the ASAS lightcurve is obvious, with small mean photometric errors with respect to the peak-to-peak amplitude from 10.8 to $13.0 \mathrm{mag}\left(\overline{\delta V_{0}}=0.032 \mathrm{mag}\right)$. RY Ori is one of the six identified variable stars with power spectra maxima larger 

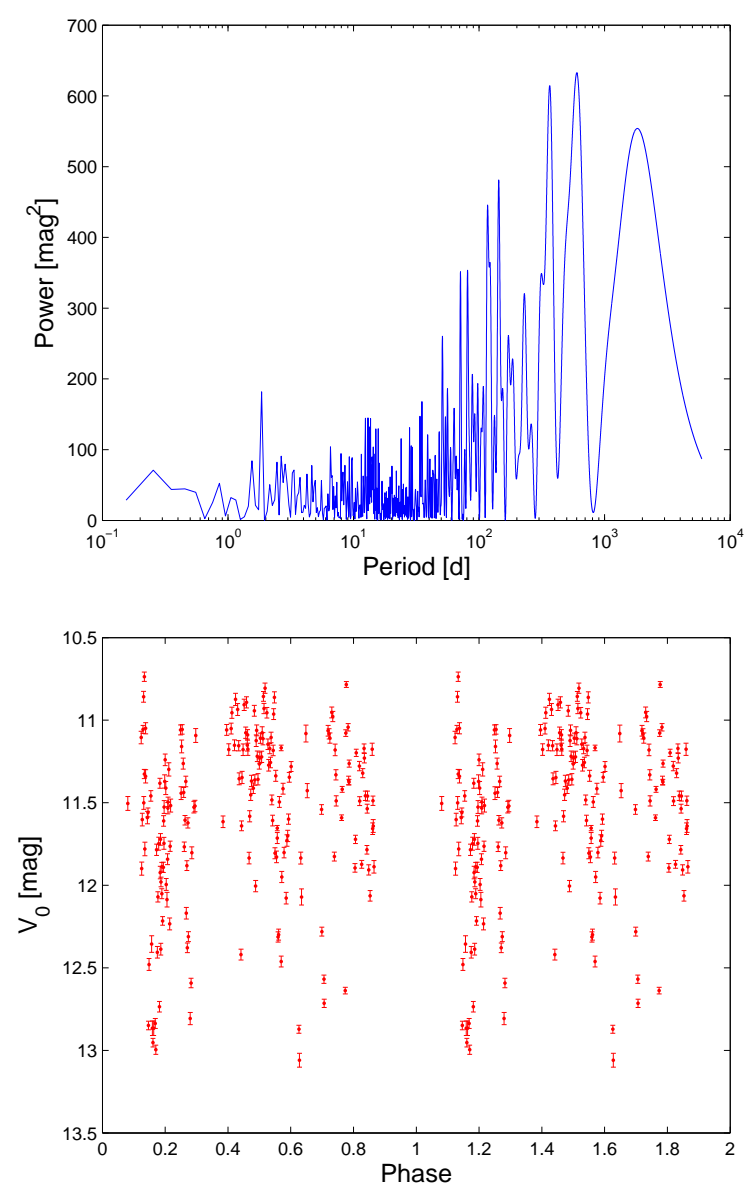

Fig. 5 Periodogram of the ASAS light curve (top) and phase-folded light curve of RY Ori to the period $P=$ $600.38 \mathrm{~d}$ (bottom).

than $500 \mathrm{mag}^{2}$ (Table 2 - actually, it is the star with the lowest power spectrum maximum). The periodogram of the ASAS light curve, displayed in Fig.5, shows no clear peak, with a number of power maxima increasing towards longer periods. The phase-folded light curve at the period of the heighest peak, at $P \sim 600 \mathrm{~d}$, does not show any evidence of real periodicity. Actually, from the full-length ligh curve in Fig. A1, the time scale of the irregular variability of RY Ori is of the order of a few days, just like many confirmed young stars in this work.

\section{TX Ori (Mayrit 521199, No. 12) and TY Ori (Mayrit 489196,}

No. 13). They are two close ( $\rho \sim 40$ arcsec $)$ pre-main sequence stars in the $\sigma$ Orionis cluster that have appeared in a number of young and emission-line star catalogues (Herbig \& Kameswara Rao 1972; Cohen \& Kuhi 1979; Herbig \& Bell 1988; Ducourant et al. 2005). As in the case of many variable stars in this work, they were discovered as variable stars by Pickering (1904). Both TX Ori and TY Ori have been subject of comprehensive studies and data compilations by Oliveira \& van Loon (2004) and Caballero (2008). Their light curves are quite similar, with minima and max- ima at about $V_{0} \sim 12.5$ and 14.0 mag. Remarkably, the TX Ori light curve displayed an apparent dimming for heliocentric Julian day 3 HJD $>3500$.

RW Ori (Mayrit 931117, No. 15). It is another member in the $\sigma$ Orionis cluster, but it is much less known than the stars above. Its photometric variability and $\mathrm{H} \alpha$ emission were first detected by Pickering (1906) and Haro \& Moreno (1953), respectively. Afterwards, it has been only investigated by Franciosini et al. (2006 - with XMM-Newton, Hernández et al. (2007 - with Spitzer), and Caballero (2008). Its ASAS light curve varied between $V_{0} \sim 12.8$ and $14.0 \mathrm{mag}$ (the outlier data point is probably unreliable).

PQ Ori (No. 21). In the discovery paper, McKnelly (1949) classified the star as a rapid, irregular variable with an observed range of variability of 12.5-13.5 mag (photographic magnitude). Although its light curve displayed many maxima and minima, he was unable to determine "any semblance of periodicity”. Herbig \& Kameswara Rao (1975), Cohen \& Kuhi (1979), and Hernández et al. (2004) measured F0, F5: and F3.0 \pm 1.5 spectral types and observed no emission lines. However, while the first authours classified it as a star in the Orion population, Hernández et al. (2004) did not find significant differences from F3 main-sequence stars nor near infrared excess, and categorized it as an object with uncertain evolutionary status. The variability patterns in the ASAS light curve of PQ Ori, although ranging between $V_{0} \sim 12.3$ and $13.3 \mathrm{mag}$, do not resemble those of other young stars in this work, and its $J-K_{\mathrm{s}}$ colour is quite blue with respect to other early $F$ stars in Ori OB1 b. As a result, its field dwarf nature seems to us more plausible.

V469 Ori (No. 36). It is an emission-line star with X-ray emission (Haro \& Moreno 1953; Wiramihardja et al. 1989; Nakano et al. 1999). According to Caballero \& Solano (2008), the star is a member in the young Ori OB1 b stellar population surrounding Alnilam, and is proably associated to the [OS98] 29J, [OS98] 29H, and [OS98] 29K remnant molecular clouds (Ogura \& Sugitani 1998). Based on its $V_{0}-K_{\mathrm{s}}$ colour and the empirical scale of effective temperatures of main-sequence stars of Alonso et al. (1996), and extrapolating it to the pre-main sequence, V469 Ori is likely a late Ge or early Ke star. Its ASAS light curve looks like those of other T Tauri stars.

V993 Ori (No. 43). It is a long-time-known variable star discovered by Luyten (1932). Caballero \& Solano (2008) classified it as a probable member of the Ori OB1 b young stellar population surrounding Alnilam. It has a very red colour $J-K_{\mathrm{s}}=1.26 \pm 0.04 \mathrm{mag}$ for its brightness $(H=$

3 The values of heliocentric Julian day used in the Figures are those tabulated by the ASAS catalogue. Actually, they are not heliocentric Julian days HJD, but the HJD minus the constant 2450000.0 (do not confuse with the constant used to transform the Julian day into the modified Julian day MJD, which is 2400000.5 ). 
$9.82 \pm 0.02 \mathrm{mag}$ ), which may indicate the presence of a circumstellar disc or, alternatively, that it is a background giant star. It may be the optical/near infrared counterpart of IRAS Z05354-0107, a source in the IRAS Faint Source Reject Catalog (Moshir 1992). There is no spectroscopy available to confirm or discard its possible $\mathrm{T}$ Tauri status. The large peak-to-peak amplitude of its light curve of $V_{0} \sim 13.0$ to fainter than $14.5 \mathrm{mag}$, although sheds light on the photometric variability of V993 Ori, is not conclusive.

GSC 04767-00071 (No. 48). Schirmer et al. (2009) classified it as a chromospherically active star suspected of variability in the ROTSE-1 database. The star GSC 04767-00071 is probably associated to the X-ray source 1RXS J053944.7005612 (Voges et al. 1999) and has a very low PPMX proper motion, of $\left(\mu_{\alpha} \cos \delta, \mu_{\delta}\right)=(-6 \pm 2,-9 \pm 2)$ mas a $^{-1}$. The optical and near-infrared photometry supports membership in Ori OB1 b. It may be a very young solar analog. The amplitude of photometric variability of its ASAS lightcurve, of $V_{0} \sim 10.8$ to $11.1 \mathrm{mag}$, is relatively small.

PU Ori (No. 51). It is a pre-main sequence star near the Alnilam supergiant with $\mathrm{H} \alpha$ in strong two-lobe emission (Haro \& Moreno 1953; Herbig \& Kameswara Rao 1972; Cohen \& Kuhi 1979; Wiramihardja et al. 1989), photometric variability (Fedorovich 1960; Briceño et al. 2005), midinfrared flux excess at the IRAS passbands (Weintraub 1990; Weaver \& Jones 1992), and forbidden emission lines ([O I] $\lambda 6300.3 \AA$; Hirth et al. 1997). It has a very red colour of $J-K_{\mathrm{s}}=1.77 \pm 0.03 \mathrm{mag}$ (Caballero \& Solano 2008). From our light curve, PU Ori is an irregular variable with minimum and maximum magnitudes $V_{0} \sim 12.5$ and $14.5 \mathrm{mag}$.

V472 Ori (No. 53). It is an $\mathrm{H} \alpha$ emitter star (Haro \& Moreno 1953; Wiramihardja et al. 1989) of known photometric variability (Fedorovich 1960; Cieslinski et al. 1997). It may be the optical/near infrared counterpart of IRAS Z05322 0039. V472 Ori is a probable member in the Mintaka cluster (Caballero \& Solano 2008). Its ASAS light curve has a peak-to-magnitude larger than 1 mag.

V351 Ori (No. 70). It is a HAeBe A7IIIe variable star of $\delta$ Scuti type discovered by Hoffmeister (1934). It has been investigated in detail, especially after Kovalchuk (1984) observed a flare-like event on its light curve at the $U B V R$ bands (Wouterloot \& Walmsley 1986; Sterken et al. 1993; Rosenbush 1995; Marconi et al. 2000; Balona et al. 2002; Vieria et al. 2003). It has been proposed that a temporarily strong accretion of matter onto the star or extinction by circumstellar dust clouds takes place to explain the remarkable V351 Ori change of behaviour from "that of a [HAeBe] star with strong photometric variations [...] to that of an almost non-variable star" (van den Ancker et al. 1996). The main period of pulsations of V351 Ori is only of the order of $0.06 \mathrm{~d}$ with a peak-to-peak amplitude of about $0.1 \mathrm{mag}$ (van den Ancker et al. 1998; Marconi et al. 2001; Ripepi et al. 2003). The pre-main sequence nature of the star is supported by the presence of $\mathrm{H} \alpha$ in emission, a pronounced inverse P Cygni profile, S I $\lambda 1296 \AA$ and O I $\lambda 1304 \AA$ lines in emission, and infrared excess (Valenti et al. 2000 and references above). With $V_{0} \approx 8.91 \mathrm{mag}$ and $20-30 \mathrm{Jy}$ at the 60 and $100 \mu \mathrm{m}$ IRAS passbands, it is by far the brightest star in our sample at all wavelengths, but also the less variable one. However, the amplitude of variability, between $V_{0} \sim$ 8.80 and $9.15 \mathrm{mag}$, is slightly larger than previously measured. It may be due to our long (ASAS) temporal coverage, probably longer than any other previous monitoring.

StHa 40 (No. 78). It was first listed in the $\mathrm{H} \alpha$-emission star catalogues of MacConnell (1982) and Stephenson (1986) and followed-up afterwards by Downes \& Keyes (1988), Torres et al. (1995), Gregorio-Hetem \& Hetem (2002), and Maheswar et al. (2003). StHa 40 is a T Tauri star with variable spectral type (from F8, through G2 and G5, to K0), variable $\mathrm{H} \alpha$ emission (from $\mathrm{pEW}(\mathrm{H} \alpha)=-14.5 \AA$ to a faint absorption), lithium absorption (from $\mathrm{pEW}(\mathrm{Li} \mathrm{I})=+0.13$ to $+0.33 \AA$ ), and radial velocity (indicative of possible spectral binarity). Besides, Gregorio-Hetem \& Hetem (2002) associated StHa 40 to a ROSAT X-ray source and derived basic stellar and circumstellar parameters. Maheswar et al. (2003) estimated a magnitude difference $\Delta V \sim 1.6 \mathrm{mag}$ between two optical spectra taken almost three years apart and after comparison with spectro-photometric standards. The $\mathrm{H} \alpha$ line was in emission [absorption] when the star was brighter, $V \sim 10.9 \mathrm{mag}$ [fainter, $V \sim 12.5 \mathrm{mag}$ ]. However, while the brightest Maheswar et al. (2003)'s estimation for $V$ agrees very well with our light curve (from were we measure $V_{0, \text { bright }} \approx 11.0 \mathrm{mag}$ ), none of the $173 \mathrm{StHa} 40$ data points covering almost six years were fainter than $V_{0, \text { faint }} \approx$ $11.8 \mathrm{mag}$.

\subsubsection{Previously unknown variable young stars}

Of the four stars in this class, only two (Mayrit $528005 \mathrm{AB}$ and StHa 48) have uncontrovertible signposts of youth. The other two stars (Kiso A-0903 135 and HD 290625) have not been well investigated yet and may not belong to the young Orion Belt stellar population.

Mayrit 528005 AB (No. 18). It is a hierarchical triple system in the $\sigma$ Orionis cluster, made of a close binary $\left(\rho_{\mathrm{A}-\mathrm{B}}=\right.$ $0.40 \pm 0.08$ arcsec) resolved with adaptive optics at $\rho_{\mathrm{AB}-\mathrm{C}} \sim$ 7.6 arcsec to the low-mass star Mayrit 530005 (S Ori J053847.5022711; Caballero 2005, 2009). The binary primary has a cosmic lithium abundance (Zapatero Osorio et al. 2002), double-peak $\mathrm{H} \alpha$ (Caballero 2006), and strong X-ray emissions (Wolk 1996; Fraciosini et al. 2006; Caballero et al. 2009), and an spectral energy distribution typical of class II objects (Hernández et al. 2007). The ASAS light curve shows variability between $V_{0} \sim 13$ and $14 \mathrm{mag}$, and an apparent series of brightenings at $\mathrm{HJD} \sim 3000$. 
Kiso A-0903 135 (No. 41). It is an emission-line star only referenced once by Kogure et al. (1989), who measured a weak $\mathrm{H} \alpha$ emission intensity and placed it in the Orion region. Its optical (DENIS; Epchtein et al. 1997) and near-infrared (2MASS) photometry and null proper motion (within error bars; USNO-B1) are consistent with membership in the Ori OB1 b association. With $\sigma_{V_{0}}=0.480 \mathrm{mag}$, it is one of the most variable stars in the study. While its brightest magnitude is $V_{0} \sim 12.8 \mathrm{mag}$, the faintest one is $V_{0} \sim 15.0$ mag or fainter.

StHa 48 (No. 55). It is a K4-type T Tauri star with $\mathrm{H} \alpha$ in emission and Li I in absorption (Stephenson 1986; Maheswar et al. 2003; Caballero \& Solano 2008). The star, which is located in the surroundings of Alnilam, has a wide amplitude of variability, with maximum and minimum brighteness at $V_{0} \approx 11.9$ and $13.3 \mathrm{mag}$, respectively, and time scales of variation of a few days.

HD 290625 (No. 74). It is an A2 star classified as a probable member in the young stellar population of Ori OB1 b (Guetter 1979; Nesterov et al. 1995). The ASAS light curve displayed variations between $V_{0} \approx 10.8$ and $11.3 \mathrm{mag}$, approximately. The Tycho-2 and 2MASS photometry and the low proper motion, of less than 4 mas a $^{-1}$ (Tycho-2, PPMX, USNO-B1), are consistent with the spectral type and a long heliocentric distance. Besides, lifes of A2 stars are relatively short, which confirm the HD 290625 youth. However, the star is a couple of magnitudes fainter than expected for "normal" A2 Orion stars at $d \sim 0.4 \mathrm{kpc}$. Instead of resorting to another young population in the far background of Orion, of which there is no evidence, we propose that HD 290625 is a variable $\mathrm{HAeBe}$ star with a scattering edge-on disc in the Orion complex, just like UX Ori or the $\sigma$ Orionis star StHa 50 (Mayrit 459340 - Thé et al. 1994; Caballero et al. 2008) or, alternatively, a bright, blue, subdwarf (there have been previous detections of such objects in the area: Caballero \& Solano 2007; Vennes et al. 2007).

\subsection{Variable background giants}

Unfortunately, because of the relative resemblance between the spectral energy distributions of distant giant stars with extended cool envelopes and of young stars with developed discs (due to the common strong infrared flux excess), giant stars sometimes contaminate lists of member candidates in star-forming regions. For example, the bright (in the infrared) giant star IRAS 05358-0238 was once classified as a Class I object in the $\sigma$ Orionis cluster (Oliveira \& van Loon 2004; Hernández et al. 2007; Caballero 2008). Since the surveyed area in this work is rich in star-forming regions (including $\sigma$ Orionis), we had to be careful with disentangling both pre-main sequence and giant star populations.

Of the eight identified variable background giants, only one (No. 60) has no IRAS excesses or were not detected with the Spatial Infrared Imaging Telescope onboard the
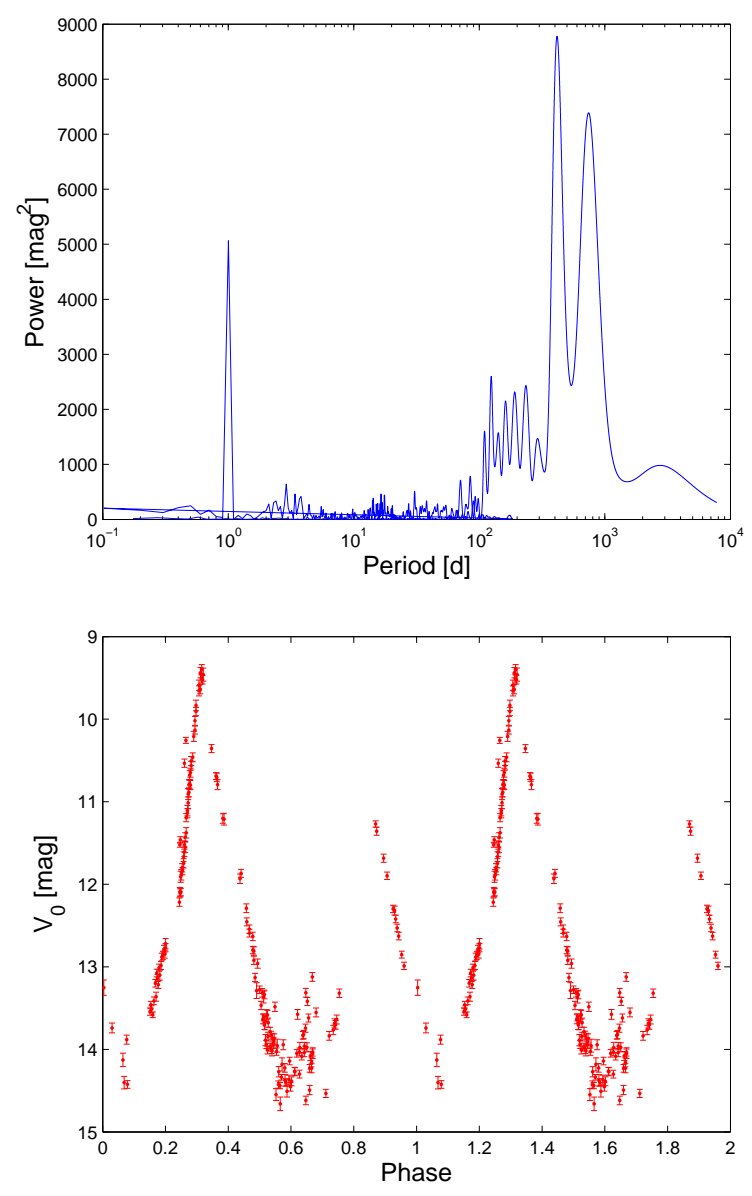

Fig. 6 Same as Fig. 5, but for $\mathrm{X}$ Ori and a period $P=$ $416.05 \mathrm{~d}$.

Midcourse Space Experiment (8.3-21.3 $\mu \mathrm{m}$; Kraemer et al. 2003). There were also only three stars previously classified as giants: X Ori, GT Ori, and IRAS 05354-0142 (for which variability had not ever been proposed). Therefore, in this work we present six new variable giants. Besides, we measure periods of photometric variability (with maxima of the power spectrum above $500 \mathrm{mag}^{2}$ ) between 78 and $920 \mathrm{~d}$ for four of the eight giants, while only one, X Ori, had been reported before to display periodicity.

\subsubsection{Known background giants}

X Ori (No. 29). This giant or superginat star was the reddest object in the Alnilam-Mintaka area studied by Caballero \& Solano (2008), but is located at about twice the heliocentric distance to the Ori OB1 association (Jura et al. 1993). It is an M8-9-type Mira Ceti variable found by Wolf (1904) with $P=424.75 \pm 1.77 \mathrm{~d}$ (Templeton et al. 2005) and silicate dust emission (Sloan \& Price 1998; Speck et al. 2000). In particular, the 9.7 and $18 \mu \mathrm{m}$ silicate dust feature originates from the circumstellar envelopes of oxygenrich asymptotic giant branch stars (Kwok et al. 1997). From our periodogram (Fig. 6), there are two clear peaks at $P \approx$ 

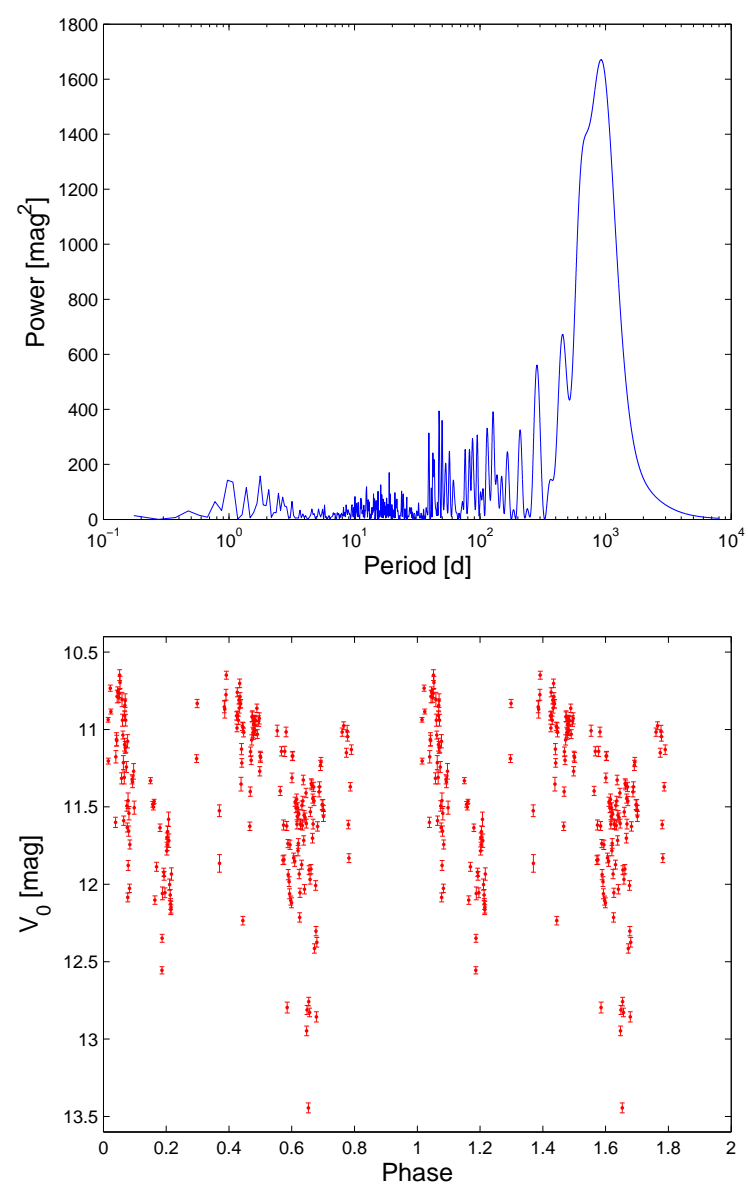

Fig. 7 Same as Fig. 5, but for GT Ori and a period $P=$ 920.32 d.

$416 \mathrm{~d}$, consistent with literature values assuming a reasobale error of $2 \%$, and its harmonic at $P \approx 832 \mathrm{~d}$. There is also a spurious (systematic) peak at $P=1 \mathrm{~d}$. The triangular shape of minima and maxima in the phase-folded light curve may be useful for modelling the astrophysical properties of the Mira star.

GT Ori (No. 66). It is a semi-regular pulsating star, originally proposed to be a likely Mira star with variations between 11.5 and 12.5 mag by Hoffmeister (1934). Afterwards, it has been monitored by several authors (e.g. Kurochkin 1949; Braune 2001). The F0 spectral type determined by Nesterov et al. (1995) is in contradiction with the red optical and near-infrared colours, the detection of GT Ori by $I R A S$ at the 12 and $25 \mu \mathrm{m}$, and its low proper motion, of less than 3 mas a $^{-1}$. The photometric and astrometric information and the strong periodogram peak at $P \sim 920 \mathrm{~d}$ and the phase-folded light curve in Fig. 7 are more consistent with the semi-regular pulsating status and a probable K-type, giant nature of GT Ori. The large amplitude of variability of the star, of almost $3 \mathrm{mag}\left(V_{0} \approx 10.6-13.5 \mathrm{mag}\right)$, and the overlapping of different (shorter) periods in the light curve
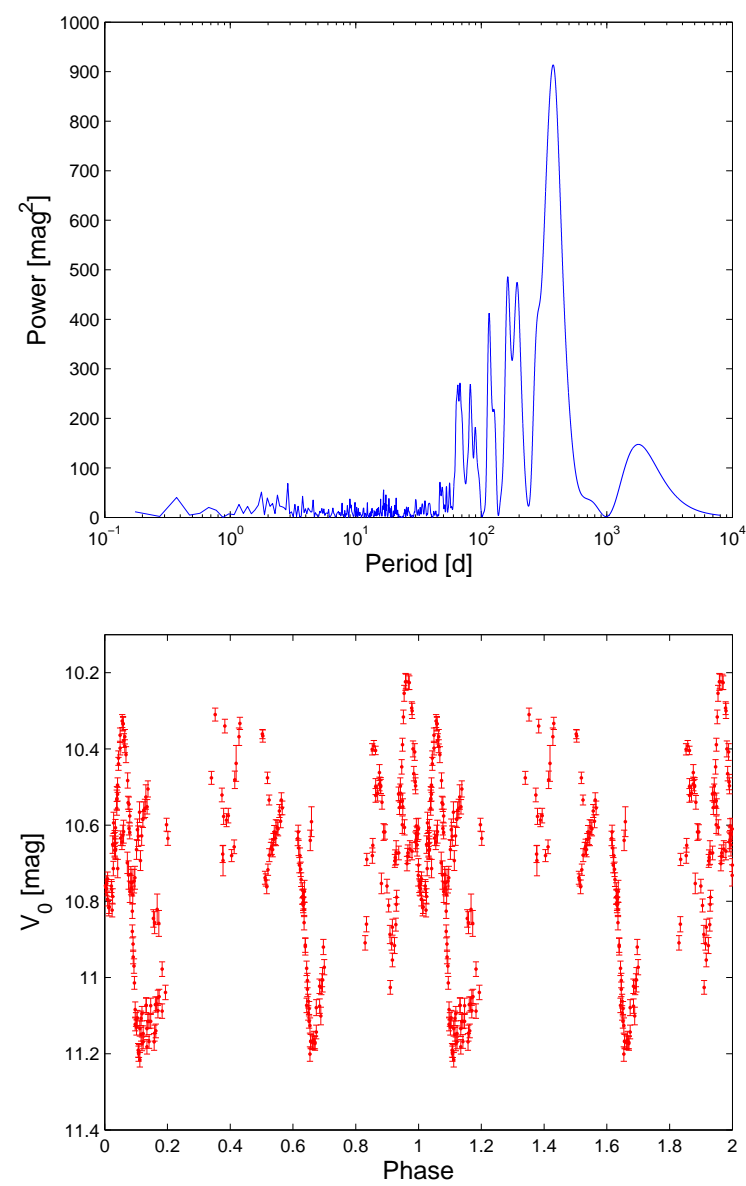

Fig. 8 Same as Fig. 5, but for IRAS 05354-0142 and a period $P=373.31 \mathrm{~d}$.

are remarkable, as well as its abnormally blue $V_{0}-J$ colour for its red $J-K_{\mathrm{s}}$ and IRAS flux excess emission.

\subsubsection{New variable giant candidates}

IRAS 05354-0142 (No. 30). With $V_{T}-K_{\mathrm{S}}=7.1 \pm 0.3 \mathrm{mag}$, it had the reddest optical-infrared colour among the $\sim 1500$ Tycho-2/2MASS stars investigated by Caballero \& Solano (2008). According to them, "the closeness of IRAS 05340142 to the Ori I-2 globule may explain part of its reddening, but not all. It might be an S-type or a C-type giant with a very late spectral type and very low effective temperature". From our ASAS data, IRAS 05354-0142 is the brightest star in Table $2\left(V_{0} \approx 10.2-11.2 \mathrm{mag}\right)$ and has the fourth largest power spectrum. Besides, it is one the four most clearly variable stars in this study, together with X Ori, RY Ori, and GT Ori. The light curve power spectrum peaks at $P=373.31 \mathrm{~d}$, but there are also secondary maxima at shorter periods (Fig. 8). The multi-pattern, phasefolded light curve indicates that a harmonic study is necessary to disentangle the different oscillation modes of the giant. At about 3 arcmin to the north lies the irregular variable star V1299 Ori, found by Kaiser (1992). It was the only 

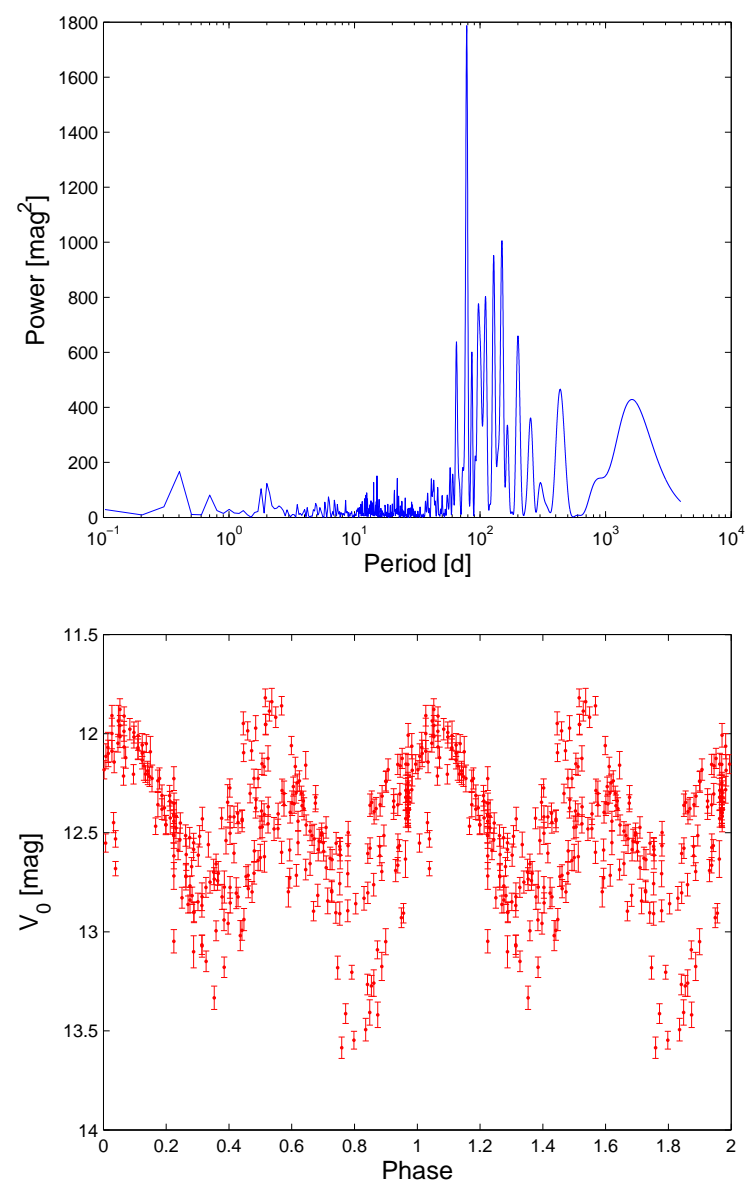

Fig. 9 Same as Fig. 5 but for IRAS 05285-0325 and a period $P=78.226 \mathrm{~d}$.

SIMBAD object unidentified by Caballero \& Solano (2008) in the Alnilam/Mintaka region. Tabulated coordinates are likely incorrect. IRAS 05354-0142 and V1299 Ori might be the same star, but the variability type and amplitude given by Kaiser (1992) are inconsistent with the data presented here.

IRAS 05285-0325 (No. 03), IRAS 05353-0309 (No. 07), IRAS 05248-0037 (No. 54), and IRAS 05312+0053 (No. 75).

They had been only reported by Kraemer et al. (2003) as "infrared" sources and have "high quality" flux densities at the IRAS $12 \mu \mathrm{m}$ (and $25 \mu \mathrm{m}$ ) pass-bands and proper motion absolute values of a few milliarcseconds per year. Of them, only one had a power spectra peak larger than $500 \mathrm{mag}^{2}$ (Table 2). The periodogram of IRAS 05285-0325 shows a forest of peaks associated to the different pulsation modes of the giant. The main mode, which is quite clear in Fig. 9 corresponds to a period $P=78.3 \pm 0.1 \mathrm{~d}$.

No. 60. It is reported here for the first time and has not a measured IRAS flux density. However, its very red optical and near-infrared colours, which puts the star in the giant
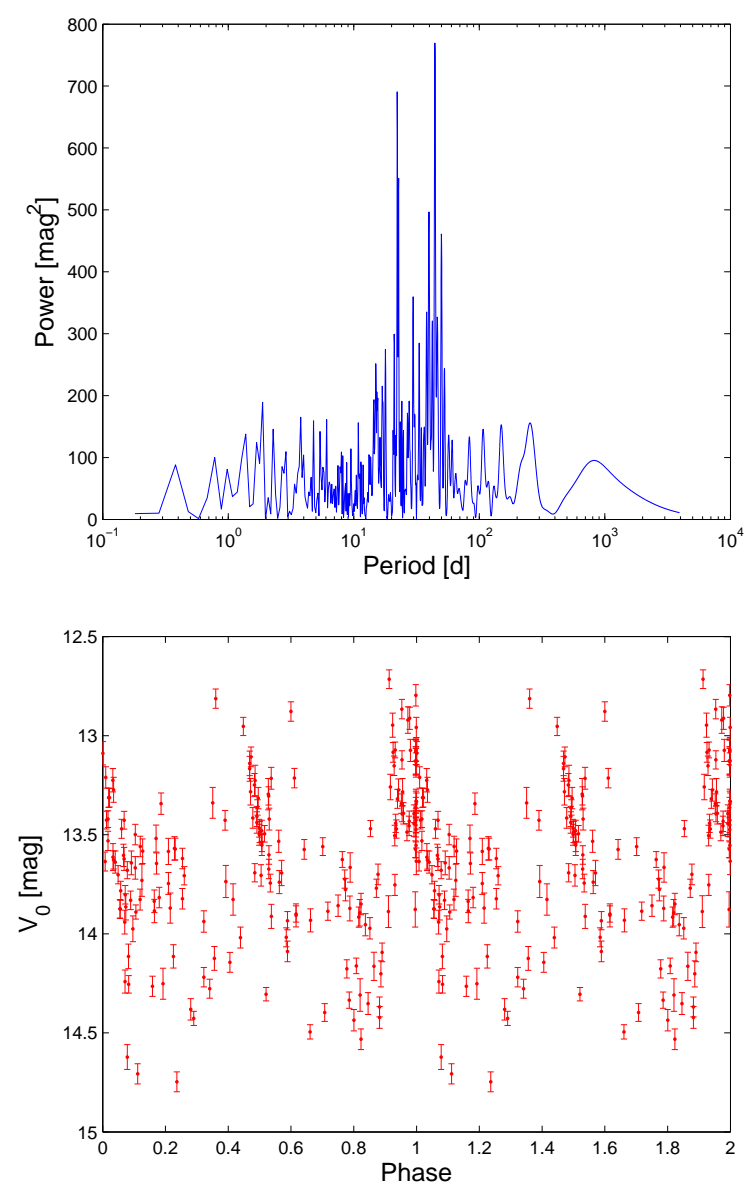

Fig. 10 Same as Fig.5 but for V1159 Ori and a period $P$ $=44.345 \mathrm{~d}$.

locus in Fig. 4 ( $V_{0}-J \sim 4.8 \mathrm{mag}$ ), and very low proper motions make No. 60 to be a fair giant candidate.

\subsection{Miscellanea}

In this class, we include variable objects that are neither young stars nor background giants. Of the eight objects in this class, only two (the dwarf nova cataclysmic variable V1159 Ori and the contact binary 2M054354-0243.6) were known to vary. At least three of the other six stars, including the nearby early F-type star HD 290509, might be eclipsing or interacting binaries.

V1159 Ori (No. 01). It is a well-known dwarf nova cataclysmic variable of ER UMa (or RZ LMi) type, which is a subgroup of SU UMa-type dwarf novad 4 with short recurrence time of super-outburst $(\tau \leq 45 \mathrm{~d})$. Their super-

\footnotetext{
4 A dwarf nova is a close binary star system in which one of the components is a white dwarf, which accretes matter from its companion. In contrast to classical novae, outbursts and super-outbursts in dwarf novae result from instabilities in the accretion disc. SU UMa dwarf novae are characterised by two distinct types of outbursts in time scales of days (more frequent) and weeks (less frequent and with larger amplitude).
} 
cycles are stable both in length and outburst pattern (Osaki 1996; Patterson 1998; Downes et al. 2001). V1159 Ori was discovered by Wolf \& Wolf (1906), who gave it the name "Var.,Orionis 36.1906", and first investigated by Kippenhahn (1953) and Jablonski \& Cieslinski (1992). Afterwards, its very short outburst cycle, superhumps, orbital periods, and high energy (X-ray, ultraviolet) emission have been studied by a number of authors (Nogami et al. 1995; Robertson et al. 1995; Patterson et al. 1995; Thorstensen et al. 1997; Szkody et al. 1999). We can add little to the knowledge on V1159 Ori except for its supercycle period. In our periodogram (Fig. 10), we detect a clear peak (and its harmonics) at $P=44.3 \pm 0.1 \mathrm{~d}$, which is agreement with, or may improve or complement, previous determinations at 44.2-46.8d (Robertson et al. 1995; Richter 1995; Kato 2001; Pitts et al. 2002).

2M054354-0243.6 ([GGM2006] 12390250, No. 14). It is a contact binary discovered by Gettel et al. (2006), who measured an heliocentric distance of $d=288 \pm 5 \mathrm{pc}$. Given the appreciable proper motion tabulated in the USNO-B1 and PPMX catalogues, $\mu=46$ mas a $^{-1}$, one can measure a transversal velocity of about $63 \mathrm{~km} \mathrm{~s}^{-1}$, which is typical of Galactic disc stars. In our periodogram (not shown here), there are two faint peaks at 0.29 and $2.3 \mathrm{~d}$, but nothing at the $0.438118 \mathrm{~d}$ measured by Gettel et al. (2006). Besides, the phase-folded light curve of 2M054354-0243.6 at this period does not show any clear periodic behaviour.

No. 33 and No. 65. They are reported here for the first time. The stars display optical and near-infrared colours typical of early $\mathrm{K}$ dwarfs and have no infrared excess. The light curve magnitudes varied between $V_{0} \approx 12.4$ and 13.4$13.6 \mathrm{mag}$ (No. 33) and $V_{0} \approx 11.7$ and $12.4 \mathrm{mag}$ (No. 65), with most of the data points concentrated towards the brightest limits. This fact may be an indication that the stars are possibly new eclisping or interacting binaries, but the possibility of being stars with magnetic spots should not be ruled out. That the periodograms (not shown here) do not display any strong peak puts a limit on the maximum periods, which are possibly shorter than a few days.

HD 290509 (No. 46). It is an anodyne F0-type Tycho-2 star with only one entry in SIMBAD (Nesterov et al. 1995). The appreciable proper motion tabulated by PPMX, of ( $30.6 \pm 1.3$ $-8.8 \pm 1.3)$ mas a $^{-1}$, indicates that it is a field star and not a young star in Orion. Its optical and red colours match those of dwarfs of the same spectral type. The ASAS light curve of HD 290509 resembles that of the star No. 33, but with a shorter range of variation, between $V_{0} \approx 10.35$ and 10.60 mag. It might be another short-period eclipsing binary.

VSS VI-32 (No. 72). It is an unpolarised star in the background of the Messier 78 star-forming complex, only reported by Vrba et al. (1976). Its very red colours, of $V_{0}-$
$J \sim 2.5 \mathrm{mag}$ and $J-K_{\mathrm{s}}=1.49 \pm 0.03 \mathrm{mag}$, may not be intrinsic, but due to the high extinction in the area. Its variability class is unknown to us.

No. 06, No. 73. They are also reported here for the first time and none of them have a measured IRAS flux density. They have very low proper motions and are the reddest (in $J-K_{\mathrm{s}}$ ) stars in this section except for VSS VI-32, but do not seem to be red enough to be classified as giant star candidates. Their light curves are quite different, with No. 06's one varying only between 11.05 and $11.45 \mathrm{mag}$, and No. 73's one between 12.0 and $14.0 \mathrm{mag}$. Neither the power spectrum analysis nor the light curve shapes provide any clear indication of the nature of the two stars.

\section{Summary}

With the aim of discovering and confirming variable Herbig Ae/Be and T Tauri stars among the young stellar population of the Orion Belt, we have analysed over 60000 light curves from the ASAS-3 Photometric $V$-band Catalogue of the All Sky Automated Survey in a squared area of side 5 deg centred on the supergiant star Alnilam ( $\epsilon$ Ori, the middle star of the Orion Belt).

After consecutive filterings, visual inspections with the virtual observatory tool Aladin, and cross-matches with infrared and astrometric catalogues, we kept 32 variable stars with average magnitudes $\overline{V_{0}}=8.5-13.5 \mathrm{mag}$ for a detailed analysis and data compilation. They are the most variable stars in the Orion Belt and complement the results in Pojmański (2002). Based on spectroscopic, photometric, and astrometric information from catalogues and the literature, we classified the 32 stars into:

- previously known variable young stars and candidates (12 stars). The list includes well-investigated Herbig Ae/Be| and T Tauri stars surrounding the early-type stars $\sigma$ Ori, Alnilam, and Mintaka in the Ori OB1 b association. We confirm the variable status of two active stars suspected of variability (GSC 04767-00071 and StHa 40) and mea-I sure a peak of the power spectrum larger than $500 \mathrm{mag}^{2}$ in the light curve of the emission-line, UX Ori-type variable, disc-host star RY Ori. However, the corresponding period of photometric variability seems unreliable. Thus, the 12 stars appear to be irregular variables;

previously unknown variable young stars (4 stars). They are two $\mathrm{T}$ Tauri stars with $\mathrm{Li} \mathrm{I}$ in absorption and $\mathrm{H} \alpha$ in emission (Mayrit $528005 \mathrm{AB}$ and SHa 48) and two stars earlier classified as probable members in the Ori OB1 b association. Besides, one of them, Mayrit 528005 AB, displays infrared flux excess and strong $\mathrm{X}$-ray emission, and is a close binary within a hierarchical triple system in the $\sigma$ Orionis cluster. Again, we do not find periodic trends among these young stars;

- known background giants (2 stars). The two giants (or supergiants) are X Ori, for which our period determination is consistent with those provided in the literature, 
and GT Ori, for which we firstly provide a period of photometric variability at about $920 \mathrm{~d}$ and determine abnormal optical-near infrared colours;

- new variable giant candidates (6 stars). Of them, we remark the new, extremely red, periodic variables IRAS 0535 0142 ( $P \sim 370 \mathrm{~d}$ - with a lot of shorter periods) and IRAS 05285-0325 ( $P \sim 78 \mathrm{~d})$;

- miscellanea objects (8 stars). This class contains the cataclysmic variable V1159 Ori, for which we measure a period $P=44.3 \pm 0.1 \mathrm{~d}$, in agreement with previous determinations, the contact binary 2M054354-0243.6, and six poorly investigated, new variable stars.

Some of these targets, including variable active Herbig Ae/Be and T Tauri stars in the Ori OB1 association and background giants, merit next spectroscopic analyses and photometric monitoring.

Acknowledgements. We thank M. Paegert for his helpful referee report and careful reading of the manuscript, and G. Pojmański for his kind and prompt answer to our inquiries. JAC formerly was an "investigador Juan de la Cierva" at the Universidad Complutense de Madrid and currently is an "investigador Ramón y Cajal" at the Centro de Astrobiología (CSIC-INTA). This research has made use of the SIMBAD, operated at Centre de Données astronomiques de Strasbourg, France, and the NASA's Astrophysics Data System. Financial support was provided by the Universidad Complutense de Madrid, the Comunidad Autónoma de Madrid, the Spanish Ministerio Educación y Ciencia, and the European Social Fund under grants: AyA2005-04286, AyA2005-24102-E, AyA2008-06423-C03-03, AyA2008-00695, PRICIT S-0505/ESP0237, and CSD2006-0070.

\section{References}

Acke, B., van den Ancker, M. E.: 2006, A\&A 457, 171

van den Ancker, M. E., Thé, P. S., de Winter, D.: 1996, A\&A 309, 809

van den Ancker, M. E., de Winter, D., Tjin A Djie, H. R. E.: 1998, A\&A 330, 145

Alonso, A., Arribas, S., Martínez-Roger, C.: 1996, A\&A, 313, 873

Bailer-Jones, C. A. L., Mundt, R.: 2001, A\&A 367, 218

Balona, L. A., Koen, C., van Wyk, F.: 2002, MNRAS 333, 923

Béjar, V. J. S., Zapatero Osorio, M. R., Rebolo, R.: 1999, ApJ 521, 671

Bibo, E. A., Thé, P. S.: 1991, A\&AS 89, 319

Bonnarel, F., Fernique, P., Bienaymé, O. et al.: 2000, A\&AS 143, 33

Bouvier, J., Cabrit, S., Fernández, M., Martínn, E. L., Matthews, J. M.: 1993, A\&A 272, 176

Braune, W.: 2001, Bundesdeutsche Arbeitsgemeinschaft für Veränderliche Sterne e.V. Rundbrief 50, 29

Briceño, C., Vivas, A. K., Calvet, N. et al.: 2001, Sci, 291, 93

Briceño, C., Calvet, N., Hernández, J. et al.: 2005, AJ 129, 907

Briceño, C., Hartmann, L., Hernández, J. et al.: 2007, ApJ 661, 1119

Brown, A. G. A., de Geus, E. J., de Zeeuw, P. T.: 1994, A\&A 289, 101

Caballero, J. A.: 2005, AN 326, 1007

Caballero, J. A.: 2006, PhD thesis, Universidad de La Laguna, Spain

Caballero, J. A.: 2007, A\&A 466, 917
Caballero, J. A.: 2008, A\&A 478, 667

Caballero, J. A.: 2009, Proceedings of the 15th Cambridge Workshop on Cool Stars, Stellar Systems and the Sun. AIP Conference Proceedings, 1094, 912

Cahallero, J. A., Dinis, L.: 2008, AN 329, 801

avallero, J. A., Solano, E.: 2007, ApJ 665, L151

Caballero, J. A., Solano, E.: 2008, A\&A 485, 931

Caballero, J. A., Béjar, V. J. S., Rebolo, R., Zapatero Osorio, M. R.: 2004, A\&A 424, 857

Caballero, J. A., Martín, E. L., Zapatero Osorio, M. R. et al.: 2006, A\&A 445, 143

Caballero, J. A., Béjar, V. J. S., Rebolo, R. et al.: 2007, A\&A 470, 903

Caballero, J. A., Valdivielso, L., Martín, E. L. et al.: 2008, A\&A 491,515

Caballero, J. A., López-Santiago, J., de Castro, E., Cornide, M.: 2009, AJ 137, 5012

Carpenter, J. M., Hillenbrand, L. A., Skrutskie, M. F.: 2001, AJ 121,3160

Cieslinski, D., Jablonski, F. J., Steiner, J. E.: 1997, A\&AS 124, 55

Cohen, M.: 1973, MNRAS 161, 97

Cohen, M., Kuhi, L. V.: 1979, ApJS 41, 743

Collier Cameron, A., Campbell, C. G.: 1993, A\&A 274, 309

Downes, R. A., Keyes, C. D.: 1988, AJ 96, 777

Downes, R. A., Webbink, R. F., Shara, M. M.: 2001, PASP 113, 764

Ducourant, C., Teixeira, R., Périé, J. P. et al.: 2005, A\&A 438, 769

Eiroa, C., Garzón, F., Alberdi, A. et al.: 2001, A\&A 365, 110

Eiroa, C., Oudmaijer, R. D., Davies, J. K. et al.: 2002, A\&A 384, 1038

Epchtein, N., de Batz, B., Capoani, L. et al.: 1997, Msngr 87, 27

Fedorovich, V. P.: 1960, Peremennye Zvezdy 13, 166

Franciosini, E., Pallavicini, R., Sanz-Forcada, J.: 2006, A\&A 446, 501

Gettel, S. J., Geske, M. T., McKay, T. A.: 2006, AJ 131, 621

Glass, I. S., Penston, M. V.: 1974, MNRAS 167, 237

Gregorio-Hetem, J., Hetem, A.: 2002, MNRAS 336, 197

Guetter, H. H.: 1979, AJ 84, 1846

Hamilton, C. M., Herbst, W., Shih, C., Ferro, A. J.: 2001, ApJ 554, L201

Haro, G, Moreno, A.: 1953, Bol. Obs. Tonantz. Tacub. 1, part no. 7,11

Hartmann, L., Kenyon, S. J.: 1996, ARA\&A 34, 207

Herbig, G. H.: 1977, ApJ 217, 693

Herbig, G. H., Bell, K. R.: 1988, Third catalog of emissionline stars of the Orion population, Lick Observatory Bulletin \#1111, Santa Cruz: Lick Observatory

Herbig, G. H., Kameswara Rao, N.: 1972, ApJ 174, 401

Herbst, W., Herbst, D. K., Grossman, E. J., Weinstein, D.: 1994, AJ 108, 1906

Herbst, W., Bailer-Jones, C. A. L., Mundt, R., Meisenheimer, K., Wackermann, R.: 2002, A\&A 396, 513

Hernández, J., Calvet, N., Briceño, C., Hartmann, L., Berlind, P.: 2004 AJ, 127, 1682

Hernández, J., Hartmann, L., Megeath, T. et al.: 2007, ApJ 662, 1067

Hirth, G. A., Mundt, R., Solf, J.: 1997, A\&AS 126, 437

Hoffmeister, C.: 1934, AN 253, 195

Høg, E., Fabricius, C., Makarov, V. V. et al.: 2000, A\&A 355, L27

Irwin, J., Irwin, M., Aigrain, S. et al.: 2007, MNRAS 375, 1449

Jablonski, F. J., Cieslinski, D. 1992, A\&A 259, 198

Jeffries, R. D., Maxted, P. F. L., Oliveira, J. M., Naylor, T.: 2006, MNRAS 371, L6 
Jones, B. F., Walker, M. F.: 1988, AJ 95, 1755

Joy, A. H.: 1945, ApJ 102, 168

Jura, M., Yamamoto, A., Kleinmann, S. G.: 1993, ApJ 413, 298

Kaiser, D. H.: 1992, IBVS 3814, 1

Kippenhahn, R.: 1953, AN 281, 153

Koch, R. H., Hrivnak, B. J.: 1981, ApJ 248, 249

Koenigl, A.: 1991, ApJ 370, L39

Kogure, T., Yoshida, S., Wiramihardja, S. D. et al.: 1989, PASJ 41, 1195

Kovalchuk, G. U.: 1984, Information Bulletin on Variable Stars 2482, 1

Kraemer, K. E., Shipman, R. F., Price, S. D. et al.: 2003, AJ 126, 1423

Kurochkin, N. E.: 1949, Peremennye Zvezdy 6, 303

Kwok, S., Volk, K., Bidelman, W. P.: 1997, ApJS 112, 557

Landstreet, J. D., Borra, E. F.: 1978, ApJ 224, L5

Luyten, W. J.: 1932, AN 245, 211

MacConnell, D. J.: 1982, A\&AS 48, 355

Maheswar, G., Manoj, P., Bhatt, H. C.: 2003, A\&A 402, 963

Marconi, M., Ripepi, V., Alcalá, J. M. et al.: 2000, A\&A 355, L35

Marconi, M., Ripepi, V., Bernabei, S. et al.: 2001, A\&A 372, L21

McKnelly, R. D.: 1949, AJ 55, 6

Miller Barr, J.: 1904, AN 24, 145

Monet, D. G., Levine, S. E., Canzian, B. et al.: 2003, AJ 125, 984

Mora, A., Merín, B., Solano, E. et al.: 2001, A\&A 378, 116

Moshir, M., Kopman, G., Conrow, T. A. O.: 1992, IRAS Faint Source Survey, Explanatory supplement version 2, Pasadena: Infrared Processing and Analysis Center, California Institute of Technology

Nakano, M., Yamauchi, S., Sugitani, K., Ogura, K., Kogure, T.: 1999, PASJ 51, 1

Nesterov, V. V., Kuzmin, A. V., Ashimbaeva, N. T. et al.: 1995, A\&AS 110, 367

Nogami, D., Kato, T., Masuda, S., Hirata, R.: 1995, Information Bulletin on Variable Stars 4155, 1

Ogura, K., Sugitani, K.: 1998, PASA, 15, 91

Oliveira, J. M., van Loon, J. Th.: 2004, A\&A 418, 663

Osaki, Y.: 1996, PASP 108, 39

Parihar, P., Messina, S., Distefano, E., Shantikumar N., S., Medhi, B. J.: 2009, MNRAS 400, 603

Patterson, J.: 1998, PASP 110, 1132

Patterson, J., Jablonski, F., Koen, C., O’Donoghue, D., Skillman, D. R.: 1995, PASP 107, 1183

Pickering, E. C.: 1904, AN 166, 35

Pickering, E. C.: 1906, AN 170, 317

Pojmański, G.: 1997, AcA 47, 467

Pojmański, G.: 2002, AcA 52, 397

Richter, G. A.: 1995, Astronomische Gesellschaft Abstract Series 11,149

Ripepi, V., Marconi, M., Bernabei, S. et al.: 2003, A\&A 408, 1047

Robertson, J. W., Honeycutt, R. K., Turner, G. W.: 1995, PASP 107,443

Rosenbush, A. E.: 1995, AN 316, 213

Röser, S., Schilbach, E., Schwan, H. et al.: 2008, A\&A 488, 401

Scargle, J. D.: 1982, ApJ 263, 835

Schirmer, J., Bernhard, K., Lloyd, C.: 2009, Open European Journal on Variable stars 105, 1

Scholz, A., Eislöffel, J.: 2004, A\&A 419, 249

Scholz, A., Eislöffel, J.: 2005, A\&A 429, 1007

Scholz, A., Xu, X., Jayawardhana, R. et al.: 2009, MNRAS 398, 873

Sherry, W. H., Walter, F. M., Wolk, S. J.: 2004, AJ 128, 2316

Shu, F., Najita, J., Ostriker, E. et al.: 1994, ApJ 429, 781
Skrutskie, M. F., Cutri, R. M., Stiening, R. et al.: 2006, AJ 131, 1163

Sloan, G. C., Price, S. D.: 1998, ApJS 119, 141

Speck, A. K., Barlow, M. J., Sylvester, R. J., Hofmeister, A. M.: 2000, A\&AS 146, 437

Stassun, K. G., Mathieu, R. D., Valenti, J. A.: 2006, Nature 440, 311

Stebbins, J.: 1915, ApJ 42, 133

Stephenson, C. B.: 1986, ApJ 300, 779

Sterken, C., Manfroid, J., Anton, K. et al.: 1993, ESO Sci. Rep. 12, 1

Sterzik, M. F., Alcalá, J. M., Neuhäuser, R., Schmitt, J. H. M. M.: 1995, A\&A 297, 418

Struve, O., Luyten, W. J.: 1949, ApJ 110, 160

Szkody, P., Linnell, A., Honeycutt, K. et al.: 1999, ApJ 521, 362

Templeton, M. R., Mattei, J. A., Willson, L. A.: 2005, AJ 130, 776

Terrell, D., Munari, U., Siviero, A.: 2007, MNRAS, 374, 530

Thé, P. S., de Winter, D., Pérez, M. R.: 1994, A\&AS 104, 315

Thorstensen, J. R., Taylor, C. J., Becker, C. M., Remillard, R. A.: 1997, PASP 109, 477

Torres, C. A. O., Quast, G., de La Reza, R., Gregorio-Hetem, J., Léepine, J. R. D.: 1995, AJ 109, 2146

Valenti, J. A., Johns-Krull, C. M., Linsky, J. L.: 2000, ApJS 129, 399

Vennes, S., Kawka, A., Smith, J. A.: 2007, ApJ 668, L59

Vieira, S. L. A., Corradi, W. J. B., Alencar, S. H. P. et al.: 2003, AJ 126, 2971

Voges, W., Aschenbach, B., Boller, T. et al.: 1999, A\&A 349, 389

Vrba, F. J., Strom, S. E., Strom, K. M.: 1976, AJ 81, 958

Walborn, N. R., Hesser, J. E.: 1976, ApJ, 205, L87

Walker, M. F.: 1956, ApJS 2, 365

Walker, M. F.: 1969, ApJ 155, 447

Warren, W. H., Jr., Hesser, J. E.: 1978, ApJS 36, 497

Weaver, W. B., Jones, G.: 1992, ApJS 78, 239

Weintraub, D. A.: 1990, ApJS 74, 575

Wiramihardja, S. D., Kogure, T., Yoshida, S., Ogura, K., Nakano, M.: 1989, PASJ 41, 155

Wolf, M.: 1904, AN 165, 29

Wolf, M., Wolf, G.: 1906, AN 171, 77

Wolk, S. J.: 2006, PhD thesis, State University of New York at Stony Brook, USA

Wouterloot, J. G. A., Walmsley, C. M.: 1986, A\&A 168, 237

Yudin, R. V., Evans, A.: 1998, A\&AS 131, 401

Zapatero Osorio, M. R., Béjar, V. J. S., Pavlenko, Ya. et al.: 2002, A\&A, 384, 937

Zapatero Osorio, M. R., Caballero, J. A., Béjar, V. J. S., Rebolo, R.: 2003, A\&A 408, 663

de Zeeuw, P. T., Hoogerwerf, R., de Bruijne, J. H. J., Brown, A. G. A., Blaauw, A.: 1999, AJ 117, 354

\section{A The most variable ASAS stars in the Orion Belt}



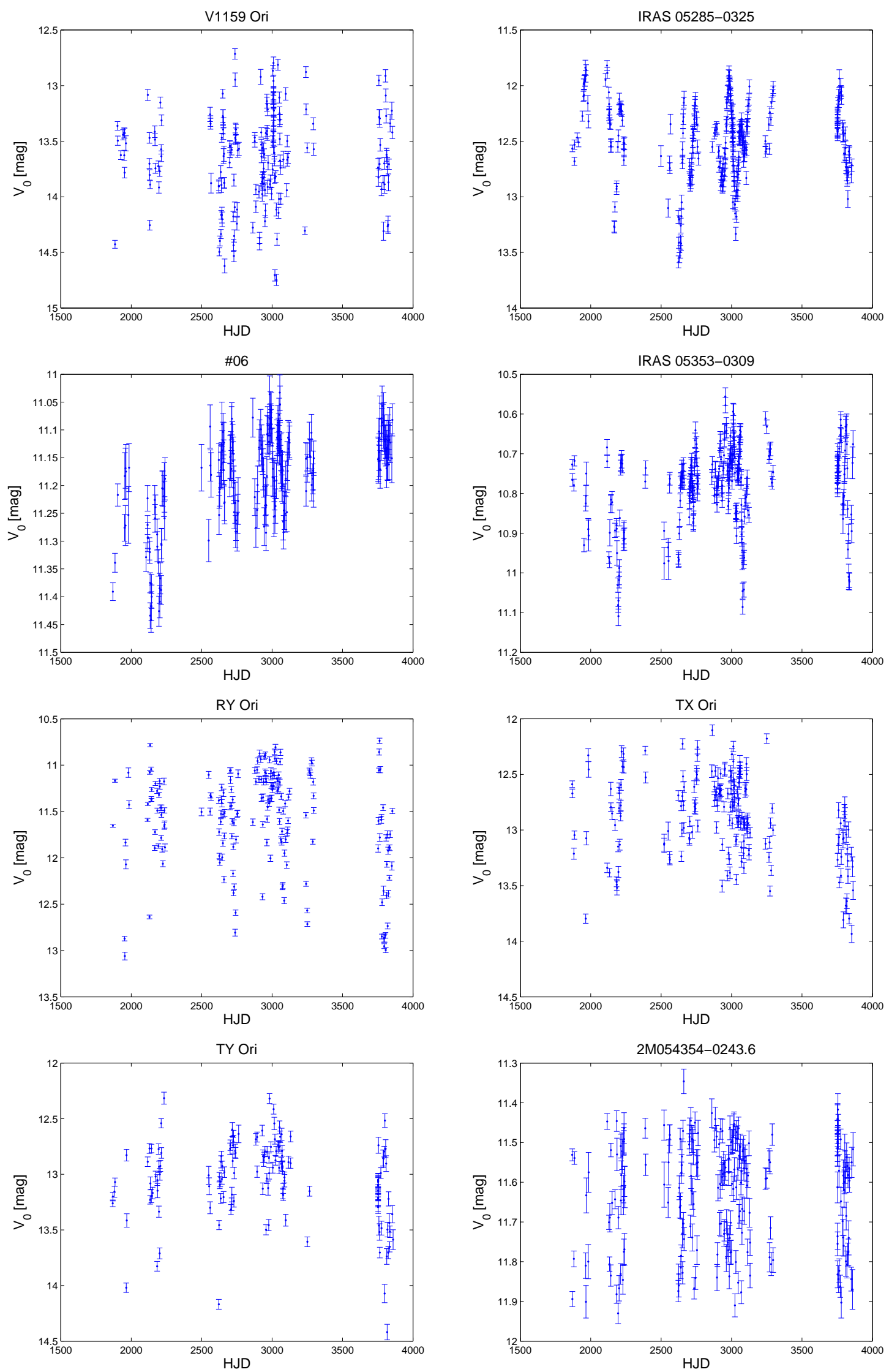

Fig. A1 ASAS ( $\left.V_{0}\right)$ light curves of the identified variables No. 01 to 14 (labeled). 

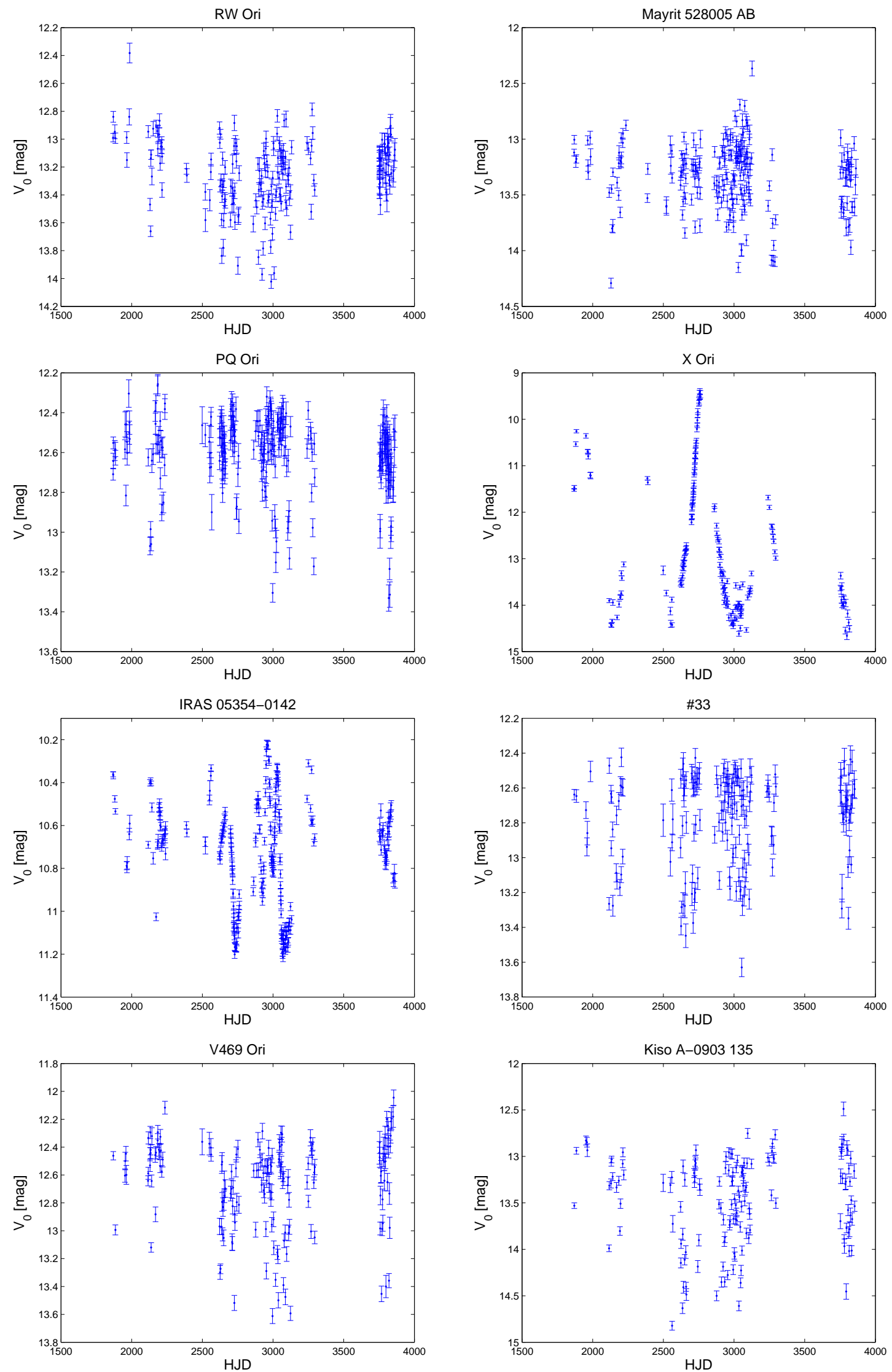

Fig. A2 Same as Fig. A1 but for identified variables No. 15 to 41. 

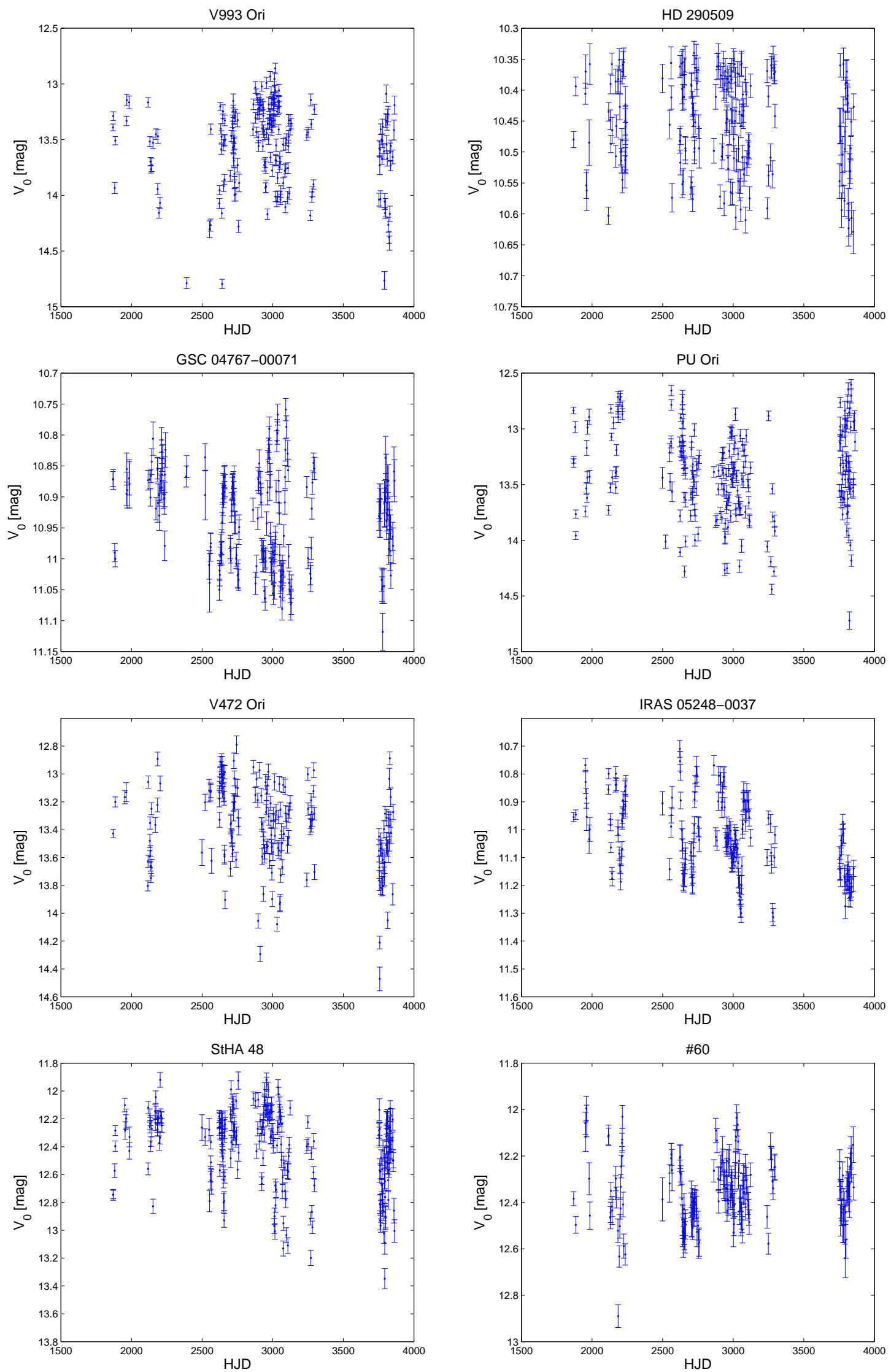

Fig. A3 Same as Fig. A1 but for identified variables No. 43 to 60 . 

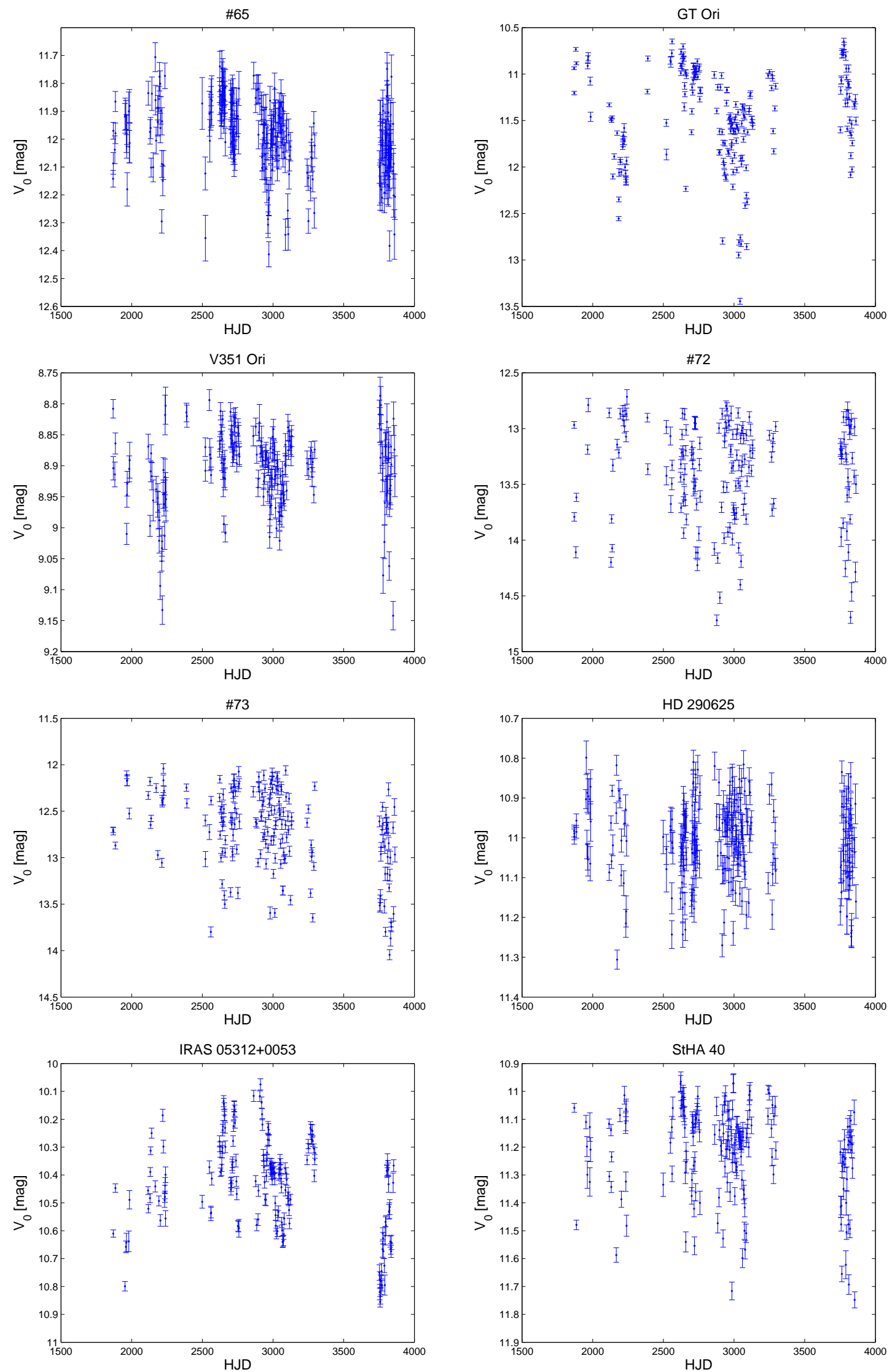

Fig. A4 Same as Fig. A1 but for identified variables No. 65 to 78 . 